\title{
TRACE FORMULAS AND A BORG-TYPE THEOREM FOR CMV OPERATORS WITH MATRIX-VALUED COEFFICIENTS
}

\author{
MAXIM ZINCHENKO
}

\begin{abstract}
We prove a general Borg-type inverse spectral result for a reflectionless unitary CMV operator (CMV for Cantero, Moral, and Velázquez [13]) associated with matrix-valued Verblunsky coefficients. More precisely, we find an explicit formula for the Verblunsky coefficients of a reflectionless CMV matrix whose spectrum consists of a connected arc on the unit circle. This extends a recent result [39] for CMV operators with scalar-valued coefficients.

In the course of deriving the Borg-type result we also use exponential Herglotz representations of Caratheodory matrix-valued functions to prove an infinite sequence of trace formulas connected with CMV operators.
\end{abstract}

\section{INTRODUCTION}

The aim of this paper is to prove a Borg-type uniqueness theorem for a special class of unitary doubly infinite block-five-diagonal matrices, that is, doubly infinite CMV matrices associated with matrix-valued Verblunsky coefficients. The actual history of CMV matrices (with scalar coefficients) is quite interesting: The corresponding unitary semi-infinite five-diagonal matrices were first introduced in 1991 by Bunse-Gerstner and Elsner [12], and subsequently discussed in detail by Watkins [76] in 1993 (cf. also the recent discussion in Simon [63]). They were subsequently rediscovered by Cantero, Moral, and Velázquez (CMV) in [13]. In [61, Sects. 4.5, 10.5], Simon introduced the corresponding notion of unitary doubly infinite five-diagonal matrices and coined the term "extended" CMV matrices. For simplicity, we will just speak of CMV operators whether or not they are half-lattice or full-lattice operators. We also note that in a context different from orthogonal polynomials on the unit circle, Bourget, Howland, and Joye [11] introduced a family of doubly infinite matrices with three sets of parameters which, for special choices of the parameters, reduces to two-sided CMV matrices on $\mathbb{Z}$. Moreover, it is possible to connect unitary block Jacobi matrices to the trigonometric moment problem (and hence to CMV matrices) as discussed by Berezansky and Dudkin [8], [9]. CMV operators with matrix-valued coefficients were recently discussed in [18], [20], [21], [63].

The relevance of CMV operators, more precisely, half-lattice CMV operators is derived from its intimate relationship with the trigonometric moment problem and hence with finite measures on the unit circle $\partial \mathbb{D}$. For a detailed account of the relationship of half-lattice CMV operators with orthogonal polynomials on the unit circle we refer to the monumental two-volume treatise by Simon [61] (see also [62],

Date: June 1, 2018.

2000 Mathematics Subject Classification. Primary 47B36, 34A55, 47A10; Secondary 34L40.

Key words and phrases. Borg-type theorem, inverse spectral problem, reflectionless, block CMV matrices, matrix-valued Verblunsky coefficients. 
[63]) and the exhaustive bibliography therein. For classical results on orthogonal polynomials on the unit circle we refer, for instance, to [6], [29]-[31], [50], [69]-[71], [74], [75]. More recent references relevant to the spectral theoretic content of this paper are [18], [26]-[28], [39], [40], [43], [52], [59], [60]. Moreover, the full-lattice CMV operators are closely related to an important, and only recently intensively studied, completely integrable nonabelian version of the defocusing nonlinear Schrödinger equation (continuous in time but discrete in space), a special case of the AblowitzLadik system. Relevant references in this context are, for instance, [1]-[5], [25], [32]-[35], [45], [51], [55]-[58], [68], [73], and the literature cited therein. We emphasize that the case of matrix-valued Verblunsky coefficients is considerably less studied than the case of scalar coefficients, but we refer to [18], [20], [21], [63].

From the outset, Borg-type theorems are inverse spectral theory assertions which typically prescribe a connected interval (or arc) as the spectrum of a self-adjoint (or unitary) differential or difference operator, and under a reflectionless condition imposed on the operator (one may think of a periodicity condition on the (potential) coefficients of the differential or difference operator) infers an explicit form of the coefficients of the operator in question. Typically, the form of the coefficients determined in this context is fairly simple and usually given by constants or functions of exponential type.

Next, we briefly describe the history of Borg-type theorems relevant to this paper. In 1946, Borg [10] proved, among a variety of other inverse spectral theorems, the following result for one-dimensional Schrödinger operators. (Throughout this paper we denote by $\sigma(\cdot)$ and $\sigma_{\text {ess }}(\cdot)$ the spectrum and essential spectrum of a densely defined closed linear operator in a complex separable Hilbert space.)

Theorem $1.1([10])$. Let $q \in L_{\text {loc }}^{1}(\mathbb{R})$ be real-valued and periodic, $H=-\frac{d^{2}}{d x^{2}}+q$ be the associated self-adjoint Schrödinger operator in $L^{2}(\mathbb{R})$, and suppose that

$$
\sigma(H)=\left[e_{0}, \infty\right) \text { for some } e_{0} \in \mathbb{R} .
$$

Then $q$ is of the form,

$$
q(x)=e_{0} \text { for a.e. } x \in \mathbb{R} .
$$

Traditionally, uniqueness results such as Theorem 1.1 are called Borg-type theorems. However, this terminology is not uniquely adopted and hence a bit unfortunate. Indeed, inverse spectral results on finite intervals in which the coefficient(s) in the underlying differential or difference expression are recovered from two spectra, were also pioneered by Borg in his celebrated paper [10], and hence are also coined Borg-type theorems in the literature, see, for instance, [53], [54].

A closer examination of the proof of Theorem 1.1 in [16] shows that periodicity of $q$ is not the point for the uniqueness result (1.2). The key ingredient (besides $\sigma(H)=\left[e_{0}, \infty\right)$ and $q$ real-valued) is the fact that

$$
\text { for all } x \in \mathbb{R}, \xi(\lambda, x)=1 / 2 \text { for a.e. } \lambda \in \sigma_{\mathrm{ess}}(H) \text {. }
$$

Here $\xi(\lambda, x)$, the argument of the boundary value $g(\lambda+i 0, x)$ of the diagonal Green's function of $H$ on the real axis (where $g(z, x)=(H-z I)^{-1}(x, x), z \in \mathbb{C} \backslash \sigma(H)$, $x \in \mathbb{R})$, is defined by

$$
\xi(\lambda, x)=\pi^{-1} \lim _{\varepsilon \downarrow 0} \operatorname{Im}(\ln (g(\lambda+i \varepsilon, x))) \text { for a.e. } \lambda \in \mathbb{R} \text { and all } x \in \mathbb{R} .
$$

Real-valued periodic potentials are known to satisfy (1.3), but so do certain classes of real-valued quasi-periodic and almost-periodic potentials $q$. In particular, 
the class of real-valued algebro-geometric finite-gap $\mathrm{KdV}$ potentials $q$ (a subclass of the set of real-valued quasi-periodic potentials) is a prime example satisfying (1.3) without necessarily being periodic. Traditionally, potentials $q$ satisfying (1.3) are called reflectionless (see [7], [15], [16], [17], and the references therein).

The extension of Borg's Theorem 1.1 to periodic matrix-valued Schrödinger operators was first proved by Dépres [23]. A new strategy of the proof based on exponential Herglotz representations and a trace formula (cf. [37]) for such potentials, as well as the extension to reflectionless matrix-valued potentials, was obtained in [16].

The direct analog of Borg's Theorem 1.1 for periodic Jacobi operators was proved by Flaschka [24] in 1975.

Theorem $1.2([24])$. Suppose $a=\left\{a_{k}\right\}_{k \in \mathbb{Z}}$ and $b=\left\{b_{k}\right\}_{k \in \mathbb{Z}}$ are periodic realvalued sequences in $\ell^{\infty}(\mathbb{Z})$ with the same period and $a_{k}>0, k \in \mathbb{Z}$. Let $H=$ $a S^{+}+a^{-} S^{-}+b$ be the associated self-adjoint Jacobi operator on $\ell^{2}(\mathbb{Z})$ and suppose that

$$
\sigma(H)=\left[E_{-}, E_{+}\right] \text {for some } E_{-}<E_{+} .
$$

Then $a=\left\{a_{k}\right\}_{k \in \mathbb{Z}}$ and $b=\left\{b_{k}\right\}_{k \in \mathbb{Z}}$ are of the form,

$$
a_{k}=\left(E_{+}-E_{-}\right) / 4, \quad b_{k}=\left(E_{-}+E_{+}\right) / 2, \quad k \in \mathbb{Z} .
$$

Here $S^{ \pm}$denote the shift operators $S^{ \pm} f=f^{ \pm}=f(\cdot \pm 1), f \in \ell^{\infty}(\mathbb{Z})$.

The extension of Theorem 1.2 to reflectionless scalar Jacobi operators is due to Teschl [66, Corollary 6.3] (see also [67, Corollary 8.6]). The extension of Theorem 1.2 to matrix-valued reflectionless Jacobi operators (and a corresponding result for Dirac-type difference operators) has recently been obtained in [17].

The first analog of Borg-type theorem for CMV operators with periodic scalar coefficients and spectrum filling out the whole unit circle was obtained by Simon in [61, Sect. 11.14]. It was recently extended in [39] to reflectionless CMV operators with scalar Verblunsky coefficients and spectra given by a connected arc on the unit circle:

Theorem 1.3 ([39]). Let $\alpha=\left\{\alpha_{k}\right\}_{k \in \mathbb{Z}} \subset \mathbb{D}$ denote Verblunsky coefficients associated with a reflectionless $C M V$ operator $\mathbb{U}(c f .(2.3)-(2.10))$ on $\ell^{2}(\mathbb{Z})$. Suppose that the spectrum of $\mathbb{U}$ consists of a connected arc on $\partial \mathbb{D}$,

$$
\sigma(\mathbb{U})=\operatorname{Arc}\left(\left[e^{i \theta_{0}}, e^{i \theta_{1}}\right]\right), \quad \theta_{0} \in[0,2 \pi), \theta_{0}<\theta_{1} \leq \theta_{0}+2 \pi .
$$

Then $\alpha=\left\{\alpha_{k}\right\}_{k \in \mathbb{Z}}$ is of the form,

$$
\alpha_{k}=\alpha_{0} g^{k}, \quad k \in \mathbb{Z}
$$

where

$$
g=-\exp \left(i\left(\theta_{0}+\theta_{1}\right) / 2\right) \text { and }\left|\alpha_{0}\right|=\cos \left(\left(\theta_{1}-\theta_{0}\right) / 4\right) .
$$

The main goal of this paper is to extend Theorem 1.3 to CMV operators with matrix-valued Verblunsky coefficients, introduced in [18]. Our study will be undertaken in the spirit of [7], [15], [16], [17], where Borg-type theorems were proven for matrix-valued Schrödinger and Dirac-type operators on $\mathbb{R}$ and similarly for matrixvalued Jacobi operators on $\mathbb{Z}$.

In Section 2 we prove an infinite sequence of trace formulas connected with CMV operators using Weyl-Titchmarsh $m$-functions (and their exponential Herglotz representations). The notion of reflectionless CMV operators is introduced in Section 3 and a variety of necessary conditions (many of them also sufficient) for a CMV 
operator to be reflectionless are established. In Section 4 we prove our main new result, a Borg-type theorem for reflectionless CMV operators with matrix-valued Verblunsky coefficients whose spectrum consists of a connected arc on the unit circle $\partial \mathbb{D}$.

\section{Trace Formulas}

In this section we introduce CMV operators with matrix-valued Verblunsky coefficients, review some basic facts on the Weyl-Titchmarsh theory associated with these operators, and derive an infinite sequence of trace formulas. We freely use the notation established in Appendix A.

Let $\ell^{2}(\mathbb{Z})^{m}=\ell^{2}(\mathbb{Z}) \otimes \mathbb{C}^{m}$ be the Hilbert space of sequences of $m$-dimensional complex-valued vectors with scalar product given by

$$
(\phi, \psi)_{\ell^{2}(\mathbb{Z})^{m}}=\sum_{k=-\infty}^{\infty} \sum_{j=1}^{m} \overline{(\phi(k))_{j}}(\psi(k))_{j}, \quad \phi, \psi \in \ell^{2}(\mathbb{Z})^{m},
$$

where we used the following notation for elements of $\ell^{2}(\mathbb{Z})^{m}$

$$
\phi=\{\phi(k)\}_{k \in \mathbb{Z}}=\left(\begin{array}{c}
\vdots \\
\phi(-1) \\
\phi(0) \\
\phi(1) \\
\vdots
\end{array}\right) \in \ell^{2}(\mathbb{Z})^{m}, \quad \phi(k)=\left(\begin{array}{c}
(\phi(k))_{1} \\
(\phi(k))_{2} \\
\vdots \\
(\phi(k))_{m}
\end{array}\right) \in \mathbb{C}^{m}, k \in \mathbb{Z} .
$$

A straightforward modification of the above definitions also yields the Hilbert space $\ell^{2}(J)^{m}$ for any $J \subset \mathbb{Z}$. For simplicity, we will abbreviate the $m \times m$ identity matrix by $I_{m}$ and the identity operator on $\ell^{2}(J)^{m}, J \subseteq \mathbb{Z}$, by $I$ without separately indicating its dependence on $J$ or $m$.

We start by introducing our basic assumption:

Hypothesis 2.1. Let $m \in \mathbb{N}$ and assume $\alpha=\left\{\alpha_{k}\right\}_{k \in \mathbb{Z}}$ is a sequence of $m \times m$ matrices with complex entries and such that

$$
\left\|\alpha_{k}\right\|_{\mathbb{C}^{m \times m}}<1, \quad k \in \mathbb{Z}
$$

Given a sequence $\alpha$ satisfying (2.3), we define two sequences of positive selfadjoint $m \times m$ matrices $\left\{\rho_{k}\right\}_{k \in \mathbb{Z}}$ and $\left\{\widetilde{\rho}_{k}\right\}_{k \in \mathbb{Z}}$ by

$$
\rho_{k}=\left(I_{m}-\alpha_{k}^{*} \alpha_{k}\right)^{1 / 2}, \quad \widetilde{\rho}_{k}=\left(I_{m}-\alpha_{k} \alpha_{k}^{*}\right)^{1 / 2}, \quad k \in \mathbb{Z} .
$$

Then (2.4) implies that $\rho_{k}$ and $\widetilde{\rho}_{k}$ are invertible matrices for all $k \in \mathbb{Z}$, and using elementary power series expansions one verifies the following identities

$$
\widetilde{\rho}_{k}^{ \pm 1} \alpha_{k}=\alpha_{k} \rho_{k}^{ \pm 1} \text { and } \alpha_{k}^{*} \widetilde{\rho}_{k}^{ \pm 1}=\rho_{k}^{ \pm 1} \alpha_{k}^{*}, \quad k \in \mathbb{Z} .
$$

According to Simon [61], we call $\alpha_{k}$ the Verblunsky coefficients in honor of Verblunsky's pioneering work in the theory of orthogonal polynomials on the unit circle [74], [75].

Next, we introduce a sequence of $2 \times 2$ block unitary matrices $\Theta_{k}$ with $m \times m$ matrix coefficients by

$$
\Theta_{k}=\left(\begin{array}{cc}
-\alpha_{k} & \widetilde{\rho}_{k} \\
\rho_{k} & \alpha_{k}^{*}
\end{array}\right), \quad k \in \mathbb{Z}
$$


and two unitary operators $\mathbb{V}$ and $\mathbb{W}$ on $\ell^{2}(\mathbb{Z})^{m}$ by their matrix representations in the standard basis of $\ell^{2}(\mathbb{Z})^{m}$ by

$$
\mathbb{V}=\left(\begin{array}{cccc}
\ddots & & & 0 \\
& \Theta_{2 k-2} & & \\
0 & \Theta_{2 k} & \\
0 & & \ddots
\end{array}\right), \mathbb{W}=\left(\begin{array}{cccc}
\ddots & & & 0 \\
& \Theta_{2 k-1} & & \\
& & \Theta_{2 k+1} & \\
0 & & \ddots
\end{array}\right),
$$

where

$$
\left(\begin{array}{cc}
\mathbb{V}_{2 k-1,2 k-1} & \mathbb{V}_{2 k-1,2 k} \\
\mathbb{V}_{2 k, 2 k-1} & \mathbb{V}_{2 k, 2 k}
\end{array}\right)=\Theta_{2 k}, \quad\left(\begin{array}{cc}
\mathbb{W}_{2 k, 2 k} & \mathbb{W}_{2 k, 2 k+1} \\
\mathbb{W}_{2 k+1,2 k} & \mathbb{W}_{2 k+1,2 k+1}
\end{array}\right)=\Theta_{2 k+1}, \quad k \in \mathbb{Z}
$$

Finally, we define the main object of our investigation, namely the unitary operator $\mathbb{U}$ on $\ell^{2}(\mathbb{Z})^{m}$ as the product of the unitary operators $\mathbb{V}$ and $\mathbb{W}$ by

$$
\mathbb{U}=\mathbb{V} \mathbb{W}
$$

or in matrix form in the standard basis of $\ell^{2}(\mathbb{Z})^{m}$, by

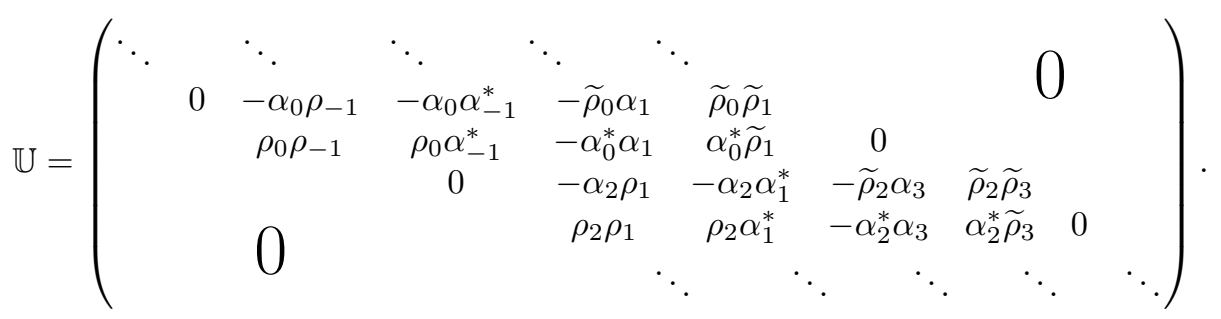

Here terms of the form $-\alpha_{2 k} \alpha_{2 k-1}^{*}$ and $-\alpha_{2 k}^{*} \alpha_{2 k+1}, k \in \mathbb{Z}$, represent the diagonal entries $\mathbb{U}_{2 k-1,2 k-1}$ and $\mathbb{U}_{2 k, 2 k}$ of the infinite matrix $\mathbb{U}$ in (2.10), respectively. We continue to call the operator $\mathbb{U}$ on $\ell^{2}(\mathbb{Z})^{m}$ the CMV operator since $(2.6)-(2.10)$ in the context of the scalar-valued semi-infinite (i.e., half-lattice) case were obtained by Cantero, Moral, and Velázquez in [13] in 2003, but we refer to the discussion in the introduction about the involved history of these operators.

Next, following [18] we recall the definition of the $2 m \times 2 m$ matrix-valued WeylTitchmarsh function $\mathcal{M}\left(\cdot, k_{0}\right), k_{0} \in \mathbb{Z}$, associated with $\mathbb{U}$,

$$
\begin{aligned}
\mathcal{M}\left(z, k_{0}\right) & =\left(\begin{array}{cc}
M_{0,0}\left(z, k_{0}\right) & M_{0,1}\left(z, k_{0}\right) \\
M_{1,0}\left(z, k_{0}\right) & M_{1,1}\left(z, k_{0}\right)
\end{array}\right) \\
& =\left(\begin{array}{cc}
\Delta_{k_{0}-1}(\mathbb{U}+z I)(\mathbb{U}-z I)^{-1} \Delta_{k_{0}-1} & \Delta_{k_{0}-1}(\mathbb{U}+z I)(\mathbb{U}-z I)^{-1} \Delta_{k_{0}} \\
\Delta_{k_{0}}(\mathbb{U}+z I)(\mathbb{U}-z I)^{-1} \Delta_{k_{0}-1} & \Delta_{k_{0}}(\mathbb{U}+z I)(\mathbb{U}-z I)^{-1} \Delta_{k_{0}}
\end{array}\right) \\
& =\oint_{\partial \mathbb{D}} d \Omega\left(\zeta, k_{0}\right) \frac{\zeta+z}{\zeta-z}, \quad z \in \mathbb{C} \backslash \partial \mathbb{D} .
\end{aligned}
$$

Here $\Delta_{k_{0}}$ denote the orthogonal projections onto the $m$-dimensional subspaces $\ell^{2}\left(\left\{k_{0}\right\}\right)^{m}, k_{0} \in \mathbb{Z}$. The nonnegative $2 m \times 2 m$ matrix-valued measure $d \Omega\left(\cdot, k_{0}\right)$, $k_{0} \in \mathbb{Z}$, is given by

$$
d \Omega\left(\zeta, k_{0}\right)=d\left(\begin{array}{ll}
\Omega_{0,0}\left(\zeta, k_{0}\right) & \Omega_{0,1}\left(\zeta, k_{0}\right) \\
\Omega_{1,0}\left(\zeta, k_{0}\right) & \Omega_{1,1}\left(\zeta, k_{0}\right)
\end{array}\right)
$$




$$
=d\left(\begin{array}{cc}
\Delta_{k_{0}-1} E_{\mathbb{U}}(\zeta) \Delta_{k_{0}-1} & \Delta_{k_{0}-1} E_{\mathbb{U}}(\zeta) \Delta_{k_{0}} \\
\Delta_{k_{0}} E_{\mathbb{U}}(\zeta) \Delta_{k_{0}-1} & \Delta_{k_{0}} E_{\mathbb{U}}(\zeta) \Delta_{k_{0}}
\end{array}\right), \quad \zeta \in \partial \mathbb{D},
$$

where $E_{\mathbb{U}}(\cdot)$ denotes the family of operator-valued spectral projections of the unitary CMV operator $\mathbb{U}$ on $\ell^{2}(\mathbb{Z})^{m}$,

$$
\mathbb{U}=\oint_{\partial \mathbb{D}} d E_{\mathbb{U}}(\zeta) \zeta
$$

It follows from Theorem A.2 that $\left.\mathcal{M}\left(\cdot, k_{0}\right)\right|_{\mathbb{D}}$ is a Caratheodory matrix and the measure $d \Omega\left(\cdot, k_{0}\right)$ can be also obtained from $\mathcal{M}\left(\cdot, k_{0}\right)$ via $(\mathrm{A} .4)$.

The Weyl-Titchmarsh function $\mathcal{M}\left(\cdot, k_{0}\right)$ is a fundamental object in the spectral theory of CMV operators. It encodes all the spectral information of the corresponding operator $\mathbb{U}$ which can be illustrated by the following result.

Theorem $2.2([18])$. The full-lattice $C M V$ operator $\mathbb{U}$ is unitarily equivalent to the operator of multiplication by $\zeta$ on $L^{2}\left(\partial \mathbb{D} ; d \Omega\left(\cdot, k_{0}\right)\right)$ for any $k_{0} \in \mathbb{Z}$. In particular,

$$
\sigma(\mathbb{U})=\operatorname{supp}\left(d \Omega\left(\cdot, k_{0}\right)\right), \quad k_{0} \in \mathbb{Z},
$$

where $\sigma(\mathbb{U})$ denotes the spectrum of $\mathbb{U}$.

We refer to Section 3 of the recent monograph [18] for a detailed discussion of this and other relations between Weyl-Titchmatsh function $\mathcal{M}\left(\cdot, k_{0}\right)$ and the associated CMV operator $\mathbb{U}$.

Next, we note that

$$
M_{0,0}\left(\cdot, k_{0}+1\right)=M_{1,1}\left(\cdot, k_{0}\right), \quad k_{0} \in \mathbb{Z}
$$

and

$$
\begin{aligned}
M_{1,1}\left(z, k_{0}\right) & =\Delta_{k_{0}}(\mathbb{U}+z I)(\mathbb{U}-z I)^{-1} \Delta_{k_{0}} \\
& =\oint_{\partial \mathbb{D}} d \Omega_{1,1}\left(\zeta, k_{0}\right) \frac{\zeta+z}{\zeta-z}, \quad z \in \mathbb{C} \backslash \partial \mathbb{D}, k_{0} \in \mathbb{Z},
\end{aligned}
$$

where

$$
d \Omega_{1,1}\left(\zeta, k_{0}\right)=d \Delta_{k_{0}} E_{\mathbb{U}}(\zeta) \Delta_{k_{0}}, \quad \zeta \in \partial \mathbb{D} .
$$

Thus, $\left.M_{0,0}\right|_{\mathbb{D}}$ and $\left.M_{1,1}\right|_{\mathbb{D}}$ are $m \times m$ Caratheodory matrices. Moreover, by (2.16) one infers that

$$
M_{1,1}\left(0, k_{0}\right)=I_{m}, \quad k_{0} \in \mathbb{Z}
$$

This implies that for any nonzero vector $x_{0} \in \mathbb{C}^{m}$ the scalar-valued Caratheodory function $m_{x_{0}}\left(z, k_{0}\right)=\left(x_{0}, M_{1,1}\left(z, k_{0}\right) x_{0}\right)_{\mathbb{C}^{m}}, z \in \mathbb{D}$, is not identically zero, and hence, $\operatorname{Re}\left(m_{x_{0}}\left(z, k_{0}\right)\right)>0$ for all $z \in \mathbb{D}$. Thus,

$$
\operatorname{Re}\left(M_{1,1}\left(z, k_{0}\right)\right)>0, \quad z \in \mathbb{D}, k_{0} \in \mathbb{Z} .
$$

It is also often beneficial to introduce the Schur matrix $\Phi_{1,1}\left(\cdot, k_{0}\right)$ associated to $M_{1,1}\left(\cdot, k_{0}\right)$ via

$$
\begin{aligned}
\Phi_{1,1}\left(z, k_{0}\right) & =\left[M_{1,1}\left(z, k_{0}\right)-I_{m}\right]\left[M_{1,1}\left(z, k_{0}\right)+I_{m}\right]^{-1}, \\
M_{1,1}\left(z, k_{0}\right) & =\left[I_{m}+\Phi_{1,1}\left(z, k_{0}\right)\right]\left[I_{m}-\Phi_{1,1}\left(z, k_{0}\right)\right]^{-1}, \quad z \in \mathbb{D} .
\end{aligned}
$$

In analogy to the exponential representation of invertible matrix-valued Herglotz functions (i.e., matrix-valued functions analytic in the open complex upper halfplane $\mathbb{C}_{+}$with nonnegative imaginary part on $\mathbb{C}_{+}$and invertible on $\mathbb{C}_{+}$, cf. [14], [38]) one obtains the following result. 
Theorem 2.3. Let $F$ be an $m \times m$ Caratheodory matrix with $F(z)$ invertible for all $z \in \mathbb{D}$. Then $-i \ln (i F)$ is an $m \times m$ Caratheodory matrix and $F$ has the exponential Herglotz representation,

$$
\begin{aligned}
& -i \ln (i F(z))=i D+\oint_{\partial \mathbb{D}} d \mu_{0}(\zeta) \Upsilon(\zeta) \frac{\zeta+z}{\zeta-z}, \quad z \in \mathbb{D}, \\
& D=-\operatorname{Re}(\ln (F(0))), \quad 0 \leq \Upsilon(\zeta) \leq \pi I_{m} \text { for } \mu_{0} \text {-a.e. } \zeta \in \partial \mathbb{D},
\end{aligned}
$$

where $d \mu_{0}$ is the normalized Lebesgue measure on $\partial \mathbb{D}(c f$. (A.7)). The $m \times m$ matrix-valued function $\Upsilon$ can be reconstructed from $F$ by

$$
\begin{aligned}
\Upsilon(\zeta) & =\lim _{r \uparrow 1} \operatorname{Re}[-i \ln (i F(r \zeta))] \\
& =(\pi / 2) I_{m}+\lim _{r \uparrow 1} \operatorname{Im}[\ln (F(r \zeta))] \text { for } \mu_{0} \text {-a.e. } \zeta \in \partial \mathbb{D} .
\end{aligned}
$$

By Theorem 2.3 and (2.18), (2.19), one then obtains the exponential Herglotz representation for $M_{1,1}\left(\cdot, k_{0}\right), k_{0} \in \mathbb{Z}$,

$$
\begin{aligned}
& -i \ln \left[i M_{1,1}\left(z, k_{0}\right)\right]=\oint_{\partial \mathbb{D}} d \mu_{0}(\zeta) \Upsilon_{1,1}\left(\zeta, k_{0}\right) \frac{\zeta+z}{\zeta-z}, \quad z \in \mathbb{D}, \\
& 0 \leq \Upsilon_{1,1}\left(\zeta, k_{0}\right) \leq \pi I_{m} \text { for } \mu_{0} \text {-a.e. } \zeta \in \partial \mathbb{D} .
\end{aligned}
$$

For our present purpose it is more convenient to rewrite (2.23) in the form

$$
\begin{aligned}
& \ln \left[M_{1,1}\left(z, k_{0}\right)\right]=i \oint_{\partial \mathbb{D}} d \mu_{0}(\zeta) \Xi_{1,1}\left(\zeta, k_{0}\right) \frac{\zeta+z}{\zeta-z}, \quad z \in \mathbb{D} \\
& -(\pi / 2) I_{m} \leq \Xi_{1,1}\left(\zeta, k_{0}\right) \leq(\pi / 2) I_{m} \text { for } \mu_{0} \text {-a.e. } \zeta \in \partial \mathbb{D},
\end{aligned}
$$

where

$$
\begin{aligned}
\Xi_{1,1}\left(\zeta, k_{0}\right) & =\lim _{r \uparrow 1} \operatorname{Im}\left[\ln \left(M_{1,1}\left(r \zeta, k_{0}\right)\right)\right] \\
& =\Upsilon_{1,1}\left(\zeta, k_{0}\right)-(\pi / 2) I_{m} \text { for } \mu_{0} \text {-a.e. } \zeta \in \partial \mathbb{D} .
\end{aligned}
$$

We note that $M_{1,1}\left(0, k_{0}\right)=I_{m}$ also implies

$$
\oint_{\partial \mathbb{D}} d \mu_{0}(\zeta) \Xi_{1,1}\left(\zeta, k_{0}\right)=0, \quad k_{0} \in \mathbb{Z}
$$

To derive trace formulas for $\mathbb{U}$ we now expand $M_{1,1}\left(z, k_{0}\right)$ near $z=0$ into a norm convergent series with matrix-valued coefficients. It follows from (2.16) that

$$
\begin{aligned}
M_{1,1}\left(z, k_{0}\right) & =\Delta_{k_{0}}(\mathbb{U}+z I)(\mathbb{U}-z I)^{-1} \Delta_{k_{0}}=I_{m}+2 z \Delta_{k_{0}} \mathbb{U}^{*}\left(I-z \mathbb{U}^{*}\right)^{-1} \Delta_{k_{0}} \\
& =I_{m}+\sum_{j=1}^{\infty} M_{j}\left(\mathbb{U}, k_{0}\right) z^{j}, \quad z \in \mathbb{D}
\end{aligned}
$$

where

$$
M_{j}\left(\mathbb{U}, k_{0}\right)=2 \Delta_{k_{0}}\left(\mathbb{U}^{*}\right)^{j} \Delta_{k_{0}}, \quad j \in \mathbb{N}, k_{0} \in \mathbb{Z} .
$$

Explicitly, using (2.10), one computes for $k_{0} \in \mathbb{Z}$,

$$
\begin{aligned}
& M_{1}\left(\mathbb{U}, k_{0}\right)=-2 \begin{cases}\alpha_{k_{0}} \alpha_{k_{0}+1}^{*}, & k_{0} \text { odd, } \\
\alpha_{k_{0}+1}^{*} \alpha_{k_{0}}, & k_{0} \text { even, }\end{cases} \\
& M_{2}\left(\mathbb{U}, k_{0}\right)
\end{aligned}
$$




$$
=2 \begin{cases}\left(\alpha_{k_{0}} \alpha_{k_{0}+1}^{*}\right)^{2}-\alpha_{k_{0}} \rho_{k_{0}+1} \alpha_{k_{0}+2}^{*} \widetilde{\rho}_{k_{0}+1}-\widetilde{\rho}_{k_{0}} \alpha_{k_{0}-1} \rho_{k_{0}} \alpha_{k_{0}+1}^{*}, & k_{0} \text { odd }, \\ \left(\alpha_{k_{0}+1}^{*} \alpha_{k_{0}}\right)^{2}-\rho_{k_{0}+1} \alpha_{k_{0}+2}^{*} \widetilde{\rho}_{k_{0}+1} \alpha_{k_{0}}-\alpha_{k_{0}+1}^{*} \widetilde{\rho}_{k_{0}} \alpha_{k_{0}-1} \rho_{k_{0}}, & k_{0} \text { even. }\end{cases}
$$

Next, we note that the Taylor expansion (2.27) implies the norm convergent expansion

$$
\ln \left(M_{1,1}\left(z, k_{0}\right)\right)=\sum_{j=1}^{\infty} L_{j}\left(\mathbb{U}, k_{0}\right) z^{j}, \quad|z| \text { sufficiently small, } k_{0} \in \mathbb{Z},
$$

where the matrix-valued coefficients $L_{j}\left(\mathbb{U}, k_{0}\right)$ can be expressed in terms of the coefficients $M_{j}\left(\mathbb{U}, k_{0}\right), j \in \mathbb{N}, k_{0} \in \mathbb{Z}$,

$$
\begin{aligned}
L_{1}\left(\mathbb{U}, k_{0}\right)= & M_{1}\left(\mathbb{U}, k_{0}\right), \\
L_{2}\left(\mathbb{U}, k_{0}\right)= & M_{2}\left(\mathbb{U}, k_{0}\right)-\frac{1}{2} M_{1}\left(\mathbb{U}, k_{0}\right)^{2}, \\
L_{3}\left(\mathbb{U}, k_{0}\right)= & M_{3}\left(\mathbb{U}, k_{0}\right)-\frac{1}{2}\left[M_{1}\left(\mathbb{U}, k_{0}\right) M_{2}\left(\mathbb{U}, k_{0}\right)+M_{2}\left(\mathbb{U}, k_{0}\right) M_{1}\left(\mathbb{U}, k_{0}\right)\right] \\
& +\frac{1}{3} M_{1}\left(\mathbb{U}, k_{0}\right)^{3}, \text { etc. }
\end{aligned}
$$

Theorem 2.4. Assume Hypothesis 2.1. Then the following trace formulas associated with the $C M V$ operator $\mathbb{U}$ hold,

$$
L_{j}\left(U, k_{0}\right)=2 i \oint_{\partial \mathbb{D}} d \mu_{0}(\zeta) \Xi_{1,1}\left(\zeta, k_{0}\right) \bar{\zeta}^{j}, \quad j \in \mathbb{N}, k_{0} \in \mathbb{Z} .
$$

In particular,

$$
\begin{aligned}
L_{1}\left(\mathbb{U}, k_{0}\right) & =-2 \begin{cases}\alpha_{k_{0}} \alpha_{k_{0}+1}^{*}, & k_{0} \text { odd } \\
\alpha_{k_{0}+1}^{*} \alpha_{k_{0}}, & k_{0} \text { even, }\end{cases} \\
& =2 i \oint_{\partial \mathbb{D}} d \mu_{0}(\zeta) \Xi_{1,1}\left(\zeta, k_{0}\right) \bar{\zeta} \\
L_{2}\left(\mathbb{U}, k_{0}\right) & =-2 \begin{cases}\alpha_{k_{0}} \rho_{k_{0}+1} \alpha_{k_{0}+2}^{*} \widetilde{\rho}_{k_{0}+1}+\widetilde{\rho}_{k_{0}} \alpha_{k_{0}-1} \rho_{k_{0}} \alpha_{k_{0}+1}^{*}, & k_{0} \text { odd }, \\
\rho_{k_{0}+1} \alpha_{k_{0}+2}^{*} \widetilde{\rho}_{k_{0}+1} \alpha_{k_{0}}+\alpha_{k_{0}+1}^{*} \widetilde{\rho}_{k_{0}} \alpha_{k_{0}-1} \rho_{k_{0}}, & k_{0} \text { even } .\end{cases} \\
& =2 i \oint_{\partial \mathbb{D}} d \mu_{0}(\zeta) \Xi_{1,1}\left(\zeta, k_{0}\right) \bar{\zeta}^{2} .
\end{aligned}
$$

Proof. Let $z \in \mathbb{D}, k_{0} \in \mathbb{Z}$. Since

$$
\frac{\zeta+z}{\zeta-z}=1+2 \sum_{j=1}^{\infty}(\bar{\zeta} z)^{j}, \quad \zeta \in \partial \mathbb{D}
$$

(2.24) implies

$$
\ln \left[M_{1,1}\left(z, k_{0}\right)\right]=2 i \sum_{j=1}^{\infty} \oint_{\partial \mathbb{D}} d \mu_{0}(\zeta) \Xi_{1,1}\left(\zeta, k_{0}\right) \bar{\zeta}^{j} z^{j}, \quad|z| \text { sufficiently small. }
$$

A comparison of coefficients of $z^{j}$ in (2.31) and (2.40) then proves (2.36). (2.37) and (2.38) follow upon substitution of (2.29) and (2.30) into (2.32) and (2.33). 


\section{Reflectionless Verblunsky Coefficients}

In this section we review basic facts about the half-lattice Weyl-Titchmarsh $m$-functions and introduce a variety of conditions for the Verblunsky coefficients $\alpha$ (resp., the CMV operator $\mathbb{U}$ ) to be reflectionless. We freely use the notation established in Appendix A.

Following the presentation of Section 2 in [18], we first recall the four fundamental $m \times m$ matrix valued sequences of Laurent polynomials $\left\{P_{+}\left(z, k, k_{0}\right)\right.$, $\left.Q_{+}\left(z, k, k_{0}\right), R_{+}\left(z, k, k_{0}\right), S_{+}\left(z, k, k_{0}\right)\right\}_{k \in \mathbb{Z}}$ associated with the CMV operator $\mathbb{U}$. These sequences are uniquely defined by the following difference relations

$$
\begin{array}{ll}
\left(\mathbb{W} P_{+}\left(z, \cdot, k_{0}\right)\right)(k)=z R_{+}\left(z, k, k_{0}\right), & \left(\mathbb{V} R_{+}\left(z, \cdot, k_{0}\right)\right)(k)=P_{+}\left(z, k, k_{0}\right), \\
\left(\mathbb{W} Q_{+}\left(z, \cdot, k_{0}\right)\right)(k)=z S_{+}\left(z, k, k_{0}\right), \quad\left(\mathbb{V} S_{+}\left(z, \cdot, k_{0}\right)\right)(k)=Q_{+}\left(z, k, k_{0}\right), \quad k \in \mathbb{Z},
\end{array}
$$

and initial conditions at some reference point $k_{0} \in \mathbb{Z}$,

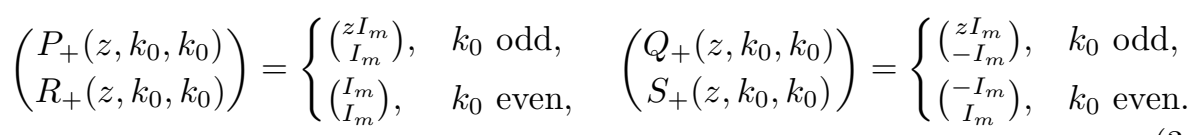

It follows that there exist unique $\mathbb{C}^{m \times m}$-valued half-lattice Weyl-Titchmarsh $m$ functions $M_{ \pm}\left(\cdot, k_{0}\right)$ such that for all $z \in \mathbb{C} \backslash(\partial \mathbb{D} \cup\{0\})$ the following $\mathbb{C}^{m \times m}$-valued sequences have square summable matrix entries, that is,

$$
\begin{aligned}
& U_{ \pm}\left(z, \cdot, k_{0}\right)=Q_{+}\left(z, \cdot, k_{0}\right)+P_{+}\left(z, \cdot, k_{0}\right) M_{ \pm}\left(z, k_{0}\right) \in \ell^{2}\left(\left[k_{0}, \pm \infty\right) \cap \mathbb{Z}\right)^{m \times m}, \\
& V_{ \pm}\left(z, \cdot, k_{0}\right)=S_{+}\left(z, \cdot, k_{0}\right)+R_{+}\left(z, \cdot, k_{0}\right) M_{ \pm}\left(z, k_{0}\right) \in \ell^{2}\left(\left[k_{0}, \pm \infty\right) \cap \mathbb{Z}\right)^{m \times m} .
\end{aligned}
$$

Moreover, one verifies that the functions $\left.M_{ \pm}\left(\cdot, k_{0}\right)\right|_{\mathbb{D}}$ are Caratheodory and antiCaratheodory matrices, respectively, and hence extend to the point $z=0$ by analyticity. In addition, the functions $M_{ \pm}$are intimately related to the half-lattice CMV operators. We refer to Section 2 in [18] for a comprehensive study of these relations.

We will call $U_{ \pm}\left(z, \cdot, k_{0}\right)$ and $V_{ \pm}\left(z, \cdot, k_{0}\right)$ the Weyl-Titchmarsh solutions associated with $\mathbb{U}$. It follows that $U_{ \pm}\left(z, \cdot, k_{0}\right)$ and $V_{ \pm}\left(z, \cdot, k_{0}\right)$ are the unique (up to rightmultiplication by constant $m \times m$ matrices) $\mathbb{C}^{m \times m}$-valued sequences that satisfy difference equations of the form (3.1) whose matrix entries are square summable near $\pm \infty$ (cf. (3.3)).

In applications it is often simpler to manipulate with Schur matrices rather than Caratheodory ones. To exploit this observation in the remainder of this section, we introduce (anti)-Schur matrices $\Phi_{ \pm}\left(\cdot, k_{0}\right)$ associated with (anti)-Caratheodory matrices $M_{ \pm}\left(\cdot, k_{0}\right)$ by

$$
\begin{aligned}
\Phi_{ \pm}\left(z, k_{0}\right) & =\left[M_{ \pm}\left(z, k_{0}\right)-I_{m}\right]\left[M_{ \pm}\left(z, k_{0}\right)+I_{m}\right]^{-1}, \\
M_{ \pm}\left(z, k_{0}\right) & =\left[I_{m}+\Phi_{ \pm}\left(z, k_{0}\right)\right]\left[I_{m}-\Phi_{ \pm}\left(z, k_{0}\right)\right]^{-1}, \quad z \in \mathbb{D} .
\end{aligned}
$$

Strictly speaking, one should always consider $\Phi_{-}^{-1}$ rather than $\Phi_{-}$as $M_{-}$is an anti-Caratheodory matrix and hence for $z \in \mathbb{D}$ the expression $\left[M_{-}\left(z, k_{0}\right)+I_{m}\right]$ is not necessarily invertible but $\left[M_{-}\left(z, k_{0}\right)-I_{m}\right]$ always is (cf. [72, p. 137]). Thus, we should have introduced the Schur matrix

$$
\Phi_{-}\left(z, k_{0}\right)^{-1}=\left[M_{-}\left(z, k_{0}\right)+I_{m}\right]\left[M_{-}\left(z, k_{0}\right)-I_{m}\right]^{-1}, \quad z \in \mathbb{D},
$$


rather than the anti-Schur matrix $\Phi_{-}$, but for simplicity of notation, we will typically avoid this complication with $\Phi_{-}$and still invoke $\Phi_{-}$rather than $\Phi_{-}^{-1}$ whenever confusions are unlikely.

Still following [18], we also mention the following two useful identities that relate the functions $\Phi_{ \pm}, \Phi_{1,1}$, and the Weyl-Titchmarsh solutions $U_{ \pm}, V_{ \pm}$to one another

$$
\Phi_{ \pm}(z, k)=\left\{\begin{array}{ll}
z V_{ \pm}\left(z, k, k_{0}\right) U_{ \pm}\left(z, k, k_{0}\right)^{-1}, & k \text { odd, } \\
U_{ \pm}\left(z, k, k_{0}\right) V_{ \pm}\left(z, k, k_{0}\right)^{-1}, & k \text { even, }
\end{array} \quad z \in \mathbb{D}, k, k_{0} \in \mathbb{Z},\right.
$$

and

$$
\Phi_{1,1}\left(z, k_{0}\right)=\left\{\begin{array}{ll}
\Phi_{-}\left(z, k_{0}\right)^{-1} \Phi_{+}\left(z, k_{0}\right), & k_{0} \text { odd, } \\
\Phi_{+}\left(z, k_{0}\right) \Phi_{-}\left(z, k_{0}\right)^{-1}, & k_{0} \text { even, }
\end{array} \quad z \in \mathbb{D}, k_{0} \in \mathbb{Z} .\right.
$$

In addition, the functions $\Phi_{ \pm}(\cdot, k)^{ \pm 1}$ satisfy the following Riccati-type equations

$$
\begin{array}{r}
\Phi_{+}(z, k) \widetilde{\rho}_{k}^{-1} \alpha_{k} \Phi_{+}(z, k-1)+z \Phi_{+}(z, k) \widetilde{\rho}_{k}^{-1}-\rho_{k}^{-1} \Phi_{+}(z, k-1)=z \rho_{k}^{-1} \alpha_{k}^{*}, \\
z \Phi_{-}(z, k)^{-1} \rho_{k}^{-1} \alpha_{k}^{*} \Phi_{-}(z, k-1)^{-1}+\Phi_{-}(z, k)^{-1} \rho_{k}^{-1}-z \widetilde{\rho}_{k}^{-1} \Phi_{-}(z, k-1)^{-1} \\
=\widetilde{\rho}_{k}^{-1} \alpha_{k}, \quad z \in \mathbb{C} \backslash \partial \mathbb{D}, k \in \mathbb{Z} .
\end{array}
$$

Next, we denote by $M_{ \pm}\left(\zeta, k_{0}\right), M_{1,1}\left(\zeta, k_{0}\right), \Phi_{ \pm}\left(\zeta, k_{0}\right)$, and $\Phi_{1,1}\left(\zeta, k_{0}\right), \zeta \in \partial \mathbb{D}$, etc., the radial limits to the unit circle of the corresponding functions,

$$
\begin{array}{ll}
M_{ \pm}\left(\zeta, k_{0}\right)=\lim _{r \uparrow 1} M_{ \pm}\left(r \zeta, k_{0}\right), & M_{1,1}\left(\zeta, k_{0}\right)=\lim _{r \uparrow 1} M_{1,1}\left(r \zeta, k_{0}\right), \\
\Phi_{ \pm}\left(\zeta, k_{0}\right)=\lim _{r \uparrow 1} \Phi_{ \pm}\left(r \zeta, k_{0}\right), & \Phi_{1,1}\left(\zeta, k_{0}\right)=\lim _{r \uparrow 1} \Phi_{1,1}\left(r \zeta, k_{0}\right), \quad \zeta \in \partial \mathbb{D}, k_{0} \in \mathbb{Z} .
\end{array}
$$

These limits are known to exist Lebesgue almost everywhere on $\partial \mathbb{D}$.

The following definition of reflectionless Verblunsky coefficients represents the analog of reflectionless coefficients in Schrödinger, Dirac, Jacobi, and CMV operators (cf., e.g. [7], [15], [16], [17] [49], in the matrix-valued coefficients context and [19], [22], [36], [37], [39], [41], [42], [44], [46]-[49], [64]-[67] in the scalar-valued coefficients context).

Definition 3.1. Assume Hypothesis 2.1 and let $\mathbb{U}$ be the associated unitary CMV operator on $\ell^{2}(\mathbb{Z})^{m}$ as defined in (2.6)-(2.10). Then $\alpha$ (resp., $\mathbb{U}$ ) is called reflectionless, if

$$
\text { for some } k_{0} \in \mathbb{Z}, M_{+}\left(\zeta, k_{0}\right)=-M_{-}\left(\zeta, k_{0}\right)^{*} \text { for } \mu_{0} \text {-a.e. } \zeta \in \sigma_{\mathrm{ess}}(\mathbb{U}) \text {. }
$$

The following result provides a variety of necessary and sufficient conditions for $\alpha$ (resp., $\mathbb{U})$ to be reflectionless.

Theorem 3.2. Let $\alpha=\left\{\alpha_{k}\right\}_{k \in \mathbb{Z}}$ satisfy Hypothesis 2.1 and $\mathbb{U}$ denote the associated unitary $C M V$ operator on $\ell^{2}(\mathbb{Z})^{m}$. Then the following assertions $(i)-(v i)$ are equivalent:

(i) $\alpha=\left\{\alpha_{k}\right\}_{k \in \mathbb{Z}}$ is reflectionless.

(ii) $\beta=\left\{\gamma_{1} \alpha_{k} \gamma_{2}^{*}\right\}_{k \in \mathbb{Z}}$ is reflectionless, where $\gamma_{1}, \gamma_{2}$ are $m \times m$ unitary matrices.

(iii) For some $k_{0} \in \mathbb{Z}, M_{+}\left(\zeta, k_{0}\right)^{*}=-M_{-}\left(\zeta, k_{0}\right)$ for $\mu_{0}$-a.e. $\zeta \in \sigma_{\mathrm{ess}}(\mathbb{U})$.

(iv) For all $k \in \mathbb{Z}, M_{+}(\zeta, k)^{*}=-M_{-}(\zeta, k)$ for $\mu_{0}$-a.e. $\zeta \in \sigma_{\text {ess }}(\mathbb{U})$.

(v) For some $k_{0} \in \mathbb{Z}, \Phi_{+}\left(\zeta, k_{0}\right)^{*}=\Phi_{-}\left(\zeta, k_{0}\right)^{-1}$ for $\mu_{0}$-a.e. $\zeta \in \sigma_{\mathrm{ess}}(\mathbb{U})$.

(vi) For all $k \in \mathbb{Z}, \Phi_{+}(\zeta, k)^{*}=\Phi_{-}(\zeta, k)^{-1}$ for $\mu_{0}$-a.e. $\zeta \in \sigma_{\text {ess }}(\mathbb{U})$.

Moreover, conditions (i)-(vi) imply the following equivalent assertions (vii)-(ix): 
(vii) For all $k \in \mathbb{Z}, M_{1,1}(\zeta, k)>0$ for $\mu_{0}$-a.e. $\zeta \in \sigma_{\text {ess }}(\mathbb{U})$.

(viii) For all $k \in \mathbb{Z},-I_{m}<\Phi_{1,1}(\zeta, k)<I_{m}$ for $\mu_{0}$-a.e. $\zeta \in \sigma_{\mathrm{ess}}(\mathbb{U})$.

(ix) For all $k \in \mathbb{Z}, \Xi_{1,1}(\zeta, k)=0$ for $\mu_{0}$-a.e. $\zeta \in \sigma_{\mathrm{ess}}(\mathbb{U})$.

Proof. We start by noting that $(i)$ is equivalent to (iii) by Definition (3.1), (iii) and $(i v)$ are equivalent to $(v)$ and $(v i)$, respectively, by (3.4), (vii) is equivalent to (viii) by (2.20), and (vii) is equivalent to (ix) by (2.25).

Next, we show that $(v)$ is equivalent to $(v i)$. One direction is trivial and for the other it suffices to check that $(v)$ at some point $k_{0}$ implies $(v)$ at points $k_{0} \pm 1$. Taking adjoint in (3.8) and solving for $\Phi_{+}\left(\zeta, k_{0}-1\right)^{*}$ one obtains

$$
\Phi_{+}\left(\zeta, k_{0}-1\right)^{*}=\left(\bar{\zeta} \widetilde{\rho}_{k_{0}}^{-1} \Phi_{+}\left(\zeta, k_{0}\right)^{*}-\bar{\zeta} \alpha_{k_{0}} \rho_{k_{0}}^{-1}\right)\left(-\alpha_{k_{0}}^{*} \widetilde{\rho}_{k_{0}}^{-1} \Phi_{+}\left(\zeta, k_{0}\right)^{*}+\rho_{k_{0}}^{-1}\right)^{-1} .
$$

Similarly, solving (3.9) for $\Phi_{-}\left(\zeta, k_{0}\right)^{-1}$ one computes

$$
\begin{aligned}
\Phi_{-}\left(\zeta, k_{0}\right)^{-1}= & \left(\zeta \widetilde{\rho}_{k_{0}}^{-1} \Phi_{-}\left(\zeta, k_{0}-1\right)^{-1}+\widetilde{\rho}_{k_{0}}^{-1} \alpha_{k_{0}}\right) \\
& \times\left(\zeta \rho_{k_{0}}^{-1} \alpha_{k_{0}}^{*} \Phi_{-}\left(\zeta, k_{0}-1\right)^{-1}+\rho_{k_{0}}^{-1}\right)^{-1} .
\end{aligned}
$$

Since by $(v) \Phi_{+}\left(\zeta, k_{0}\right)^{*}=\Phi_{-}\left(\zeta, k_{0}\right)^{-1}$ for $\mu_{0}$-a.e. $\zeta \in \sigma_{\text {ess }}(\mathbb{U})$, insertion of (3.13) into (3.12) yields

$$
\begin{aligned}
\Phi_{+}\left(\zeta, k_{0}-1\right)^{*}=( & \widetilde{\rho}_{k_{0}}^{-2} \Phi_{-}\left(\zeta, k_{0}-1\right)^{-1}+\bar{\zeta} \widetilde{\rho}_{k_{0}}^{-2} \alpha_{k_{0}} \\
& \left.-\alpha_{k_{0}} \rho_{k_{0}}^{-2} \alpha_{k_{0}}^{*} \Phi_{-}\left(\zeta, k_{0}-1\right)^{-1}-\bar{\zeta} \alpha_{k_{0}} \rho_{k_{0}}^{-2}\right) \\
\times & \left(-\zeta \alpha_{k_{0}}^{*} \widetilde{\rho}_{k_{0}}^{-2} \Phi_{-}\left(\zeta, k_{0}-1\right)^{-1}-\alpha_{k_{0}}^{*} \widetilde{\rho}_{k_{0}}^{-2} \alpha_{k_{0}}^{*}\right. \\
& \left.+\zeta \rho_{k_{0}}^{-2} \alpha_{k_{0}}^{*} \Phi_{-}\left(\zeta, k_{0}-1\right)^{-1}+\rho_{k_{0}}^{-2}\right)^{-1} \\
= & \Phi_{-}\left(\zeta, k_{0}-1\right)^{-1} \quad \text { for } \mu_{0} \text {-a.e. } \zeta \in \sigma_{\text {ess }}(\mathbb{U}) .
\end{aligned}
$$

Here (2.4) and (2.5) were used to simplify the expression. Thus, $(v)$ at $k_{0}$ implies $(v)$ at $k_{0}-1$. Similarly, one shows that $(v)$ at $k_{0}$ also implies $(v)$ at $k_{0}+1$.

Next, we verify that (iii) implies (vii). Recall that by Lemma 3.3 in [18] the resolvent $(\mathbb{U}-z I)^{-1}$ is given in terms of its matrix elements in the standard basis of $\ell^{2}(\mathbb{Z})^{m}$ by

$$
(\mathbb{U}-z I)^{-1}\left(k, k^{\prime}\right)=\frac{1}{2 z}\left\{\begin{array}{r}
U_{-}\left(z, k, k_{0}\right) W\left(z, k_{0}\right)^{-1} U_{+}\left(1 / \bar{z}, k^{\prime}, k_{0}\right)^{*} \\
k<k^{\prime} \text { or } k=k^{\prime} \text { odd } \\
U_{+}\left(z, k, k_{0}\right) W\left(z, k_{0}\right)^{-1} U_{-}\left(1 / \bar{z}, k^{\prime}, k_{0}\right)^{*} \\
k>k^{\prime} \text { or } k=k^{\prime} \text { even }
\end{array}\right.
$$

where $W\left(z, k_{0}\right)=M_{+}\left(z, k_{0}\right)-M_{-}\left(z, k_{0}\right)$ is the Wronskian of $U_{+}$and $U_{-}$. Using (iii), (3.3), (A.9), and the fact that $P_{+}\left(z, k, k_{0}\right)$ and $Q_{+}\left(z, k, k_{0}\right)$ are Laurent polynomials in $z$ and hence are analytic in $\mathbb{C} \backslash\{0\}$, one computes

$$
\begin{aligned}
\lim _{r \uparrow 1} U_{ \pm}\left(1 /(r \bar{\zeta}), k, k_{0}\right) & =\lim _{r \uparrow 1}\left[Q_{+}\left(\zeta / r, k, k_{0}\right)-P_{+}\left(\zeta / r, k, k_{0}\right) M_{ \pm}\left(r \zeta, k_{0}\right)^{*}\right] \\
& =Q_{+}\left(\zeta, k, k_{0}\right)-P_{+}\left(\zeta, k, k_{0}\right) M_{ \pm}\left(\zeta, k_{0}\right)^{*} \\
& =Q_{+}\left(\zeta, k, k_{0}\right)+P_{+}\left(\zeta, k, k_{0}\right) M_{\mp}\left(\zeta, k_{0}\right) \\
& =\lim _{r \uparrow 1} U_{\mp}\left(r \zeta, k, k_{0}\right) \text { for } \mu_{0} \text {-a.e. } \zeta \in \sigma_{\mathrm{ess}}(\mathbb{U})
\end{aligned}
$$


Thus, combining (iii), (2.16), (3.15), and (3.16) one concludes for all $k \in \mathbb{Z}$ and $\mu_{0}$-a.e. $\zeta \in \sigma_{\text {ess }}(\mathbb{U})$ that $W\left(\zeta, k_{0}\right)=M_{+}\left(\zeta, k_{0}\right)+M_{+}\left(\zeta, k_{0}\right)^{*}=W\left(\zeta, k_{0}\right)^{*}$, and hence,

$$
\begin{aligned}
M_{1,1}(\zeta, k) & =I_{m}+2 z \Delta_{k}(\mathbb{U}-z I)^{-1} \Delta_{k}=I_{m}+2 z(\mathbb{U}-z I)^{-1}(k, k) \\
& =I_{m}+ \begin{cases}U_{-}\left(\zeta, k, k_{0}\right) W\left(z, k_{0}\right)^{-1} U_{-}\left(\zeta, k, k_{0}\right)^{*}, & k \text { odd }, \\
U_{+}\left(\zeta, k, k_{0}\right) W\left(z, k_{0}\right)^{-1} U_{+}\left(\zeta, k, k_{0}\right)^{*}, & k \text { even }\end{cases}
\end{aligned}
$$

is a nonnegative Caratheodory matrix, that is, (vii) holds.

Finally, we check that $(i)$ is equivalent to $(i i)$. First, note that $\beta_{k}=\gamma_{1} \alpha_{k} \gamma_{2}^{*}$, $k \in \mathbb{Z}$, implies the following relations for matrices defined in (2.4)-(2.10) associated with Verblunsky coefficients $\alpha$ and $\beta$, respectively,

$$
\begin{aligned}
& \rho_{\beta ; k}=\gamma_{2} \rho_{\alpha ; k} \gamma_{2}^{*}, \quad \tilde{\rho}_{\beta ; k}=\gamma_{1} \widetilde{\rho}_{\alpha ; k} \gamma_{1}^{*}, \\
& \Theta_{\beta ; k}=\left(\begin{array}{cc}
\gamma_{1} & 0 \\
0 & \gamma_{2}
\end{array}\right) \Theta_{\alpha ; k}\left(\begin{array}{cc}
\gamma_{2} & 0 \\
0 & \gamma_{1}
\end{array}\right)^{*}, \quad k \in \mathbb{Z},
\end{aligned}
$$

and hence,

$$
\mathbb{V}_{\beta}=\Gamma_{1} \mathbb{V}_{\alpha} \Gamma_{2}^{*}, \quad \mathbb{W}_{\beta}=\Gamma_{2} \mathbb{W}_{\alpha} \Gamma_{1}^{*}, \quad \mathbb{U}_{\beta}=\Gamma_{1} \mathbb{U}_{\alpha} \Gamma_{1}^{*},
$$

where $\Gamma_{1}$ and $\Gamma_{2}$ are block-diagonal unitary operators on $\ell^{2}(\mathbb{Z})^{m}$ with diagonals given by

$$
\Gamma_{1}(k, k)=\left\{\begin{array}{ll}
\gamma_{1}, & k \text { odd }, \\
\gamma_{2}, & k \text { even, }
\end{array} \quad \Gamma_{2}(k, k)=\left\{\begin{array}{ll}
\gamma_{2}, & k \text { odd }, \\
\gamma_{1}, & k \text { even, }
\end{array} \quad k \in \mathbb{Z} .\right.\right.
$$

Then it follows from (3.20) and the definition of the Weyl-Titchmarsh solutions $U_{\beta ; \pm}, V_{\beta ; \pm}$ associated with the CMV operator $\mathbb{U}_{\beta}$,

$$
\begin{aligned}
& \mathbb{W}_{\beta} U_{\beta ; \pm}\left(z, \cdot, k_{0}\right)=z V_{\beta ; \pm}\left(z, \cdot, k_{0}\right), \quad \mathbb{V}_{\beta} V_{\beta ; \pm}\left(z, \cdot, k_{0}\right)=U_{\beta ; \pm}\left(z, \cdot, k_{0}\right), \\
& U_{\beta ; \pm}\left(z, \cdot, k_{0}\right), V_{\beta ; \pm}\left(z, \cdot, k_{0}\right) \in \ell^{2}\left(\left[k_{0}, \pm \infty\right) \cap \mathbb{Z}\right)^{m \times m},
\end{aligned}
$$

that the $\mathbb{C}^{m \times m}$-valued sequences $\Gamma_{1}^{*} U_{\beta ; \pm}$ and $\Gamma_{2}^{*} V_{\beta ; \pm}$ satisfy

$$
\begin{aligned}
& \mathbb{W}_{\alpha} \Gamma_{1}^{*} U_{\beta ; \pm}\left(z, \cdot, k_{0}\right)=z \Gamma_{2}^{*} V_{\beta ; \pm}\left(z, \cdot, k_{0}\right), \quad \mathbb{V}_{\alpha} \Gamma_{2}^{*} V_{\beta ; \pm}\left(z, \cdot, k_{0}\right)=\Gamma_{1}^{*} U_{\beta ; \pm}\left(z, \cdot, k_{0}\right), \\
& \Gamma_{1}^{*} U_{\beta ; \pm}\left(z, \cdot, k_{0}\right), \Gamma_{2}^{*} V_{\beta ; \pm}\left(z, \cdot, k_{0}\right) \in \ell^{2}\left(\left[k_{0}, \pm \infty\right) \cap \mathbb{Z}\right)^{m \times m} .
\end{aligned}
$$

Thus, the uniqueness of the Weyl-Titchmarsh solutions associated with $\mathbb{U}_{\alpha}$ implies that there is an $m \times m$ matrix $C$ such that

$$
\Gamma_{1}^{*} U_{\beta ; \pm}\left(z, k, k_{0}\right)=U_{\alpha ; \pm}\left(z, k, k_{0}\right) C, \quad \Gamma_{2}^{*} V_{\beta ; \pm}\left(z, k, k_{0}\right)=V_{\alpha ; \pm}\left(z, k, k_{0}\right) C,
$$

equivalently,

$$
\begin{aligned}
& U_{\alpha ; \pm}\left(z, k, k_{0}\right)= \begin{cases}\gamma_{1}^{*} U_{\beta ; \pm}\left(z, k, k_{0}\right) C, & k \text { odd }, \\
\gamma_{2}^{*} U_{\beta ; \pm}\left(z, k, k_{0}\right) C, & k \text { even, }\end{cases} \\
& V_{\alpha ; \pm}\left(z, k, k_{0}\right)=\left\{\begin{array}{ll}
\gamma_{2}^{*} V_{\beta ; \pm}\left(z, k, k_{0}\right) C, & k \text { odd, } \\
\gamma_{1}^{*} V_{\beta ; \pm}\left(z, k, k_{0}\right) C, & k \text { even, }
\end{array} \quad k \in \mathbb{Z} .\right.
\end{aligned}
$$

Inserting (3.25) and (3.26) into (3.6) we get

$$
\Phi_{\alpha ; \pm}(z, k)=\gamma_{2}^{*} \Phi_{\beta ; \pm}(z, k) \gamma_{1}, \quad z \in \mathbb{D}, k \in \mathbb{Z} .
$$


Since by $(3.20) \mathbb{U}_{\alpha}$ is unitarily equivalent to $\mathbb{U}_{\beta}$ and hence $\sigma_{\text {ess }}\left(\mathbb{U}_{\alpha}\right)=\sigma_{\text {ess }}\left(\mathbb{U}_{\beta}\right)$, we conclude from (3.27) that $\Phi_{\alpha ; \pm}(z, k)$ satisfy $(v)$ if and only if $\Phi_{\beta ; \pm}(z, k)$ do. Hence, the previously established equivalence of $(i)$ and $(v)$ finishes the proof.

It is instructive to state as a separate result the following fact obtained in the proof of Theorem 3.2 (cf. (3.20) and (3.21)).

Theorem 3.3. Let $\alpha=\left\{\alpha_{k}\right\}_{k \in \mathbb{Z}}$ be a sequence satisfying Hypothesis 2.1 and fix two $m \times m$ unitary matrices $\gamma_{1}, \gamma_{2}$. Define $\beta=\left\{\gamma_{1} \alpha_{k} \gamma_{2}^{*}\right\}_{k \in \mathbb{Z}}$. Then the $C M V$ operators $U_{\alpha}$ and $U_{\beta}$ associated with $\alpha$ and $\beta$, respectively, are unitarily equivalent.

\section{The Borg-Type Theorem for CMV Operators}

In this section we finally prove our principal new result, a general Borg-type theorem for reflectionless CMV operators with spectrum a connected subarc of the unit circle. We freely use the notation established in Appendix A.

First, we prove the following uniqueness result which is a special case of Borgtype theorem for reflectionless CMV operators.

Theorem 4.1. Let $\alpha=\left\{\alpha_{k}\right\}_{k \in \mathbb{Z}}$ be a reflectionless sequence of $m \times m$ matrix-valued Verblunsky coefficients. Let $\mathbb{U}$ be the associated unitary CMV operator (2.10) on $\ell^{2}(\mathbb{Z})^{m}$ and suppose that

$$
\sigma(\mathbb{U})=\partial \mathbb{D}
$$

Then $\alpha=\left\{\alpha_{k}\right\}_{k \in \mathbb{Z}}$ is of the form,

$$
\alpha_{k}=0, \quad k \in \mathbb{Z} .
$$

Proof. Since by hypothesis $\mathbb{U}$ is reflectionless, one infers from Definition 3.1 and Theorem 3.2 that

$$
\Phi_{+}(\zeta, k)^{*}=\Phi_{-}(\zeta, k)^{-1}, \quad \mu_{0} \text {-a.e. } \zeta \in \partial \mathbb{D}, k \in \mathbb{Z}
$$

and

$$
\Xi_{1,1}(\zeta, k)=0, \quad \mu_{0} \text {-a.e. } \zeta \in \partial \mathbb{D}, k \in \mathbb{Z} .
$$

Then it follows from (2.24) and (4.4) that $M_{1,1}(z, k)=I_{m}$ for all $z \in \mathbb{D}, k \in \mathbb{Z}$, and hence by (2.20)

$$
\Phi_{1,1}(z, k)=0, \quad z \in \mathbb{D}, k \in \mathbb{Z} .
$$

This together with (3.7) and (4.3) implies

$$
\Phi_{+}(\zeta, k)=\Phi_{-}(\zeta, k)^{-1}=0, \quad \mu_{0} \text {-a.e. } \zeta \in \partial \mathbb{D}, k \in \mathbb{Z} .
$$

Taking radial limits in the Riccati-type equations (3.8), (3.9) and substituting (4.6) into the left hand-sides yield

$$
\alpha_{k}=0, \quad k \in \mathbb{Z}
$$

Next, we introduce the following notation for closed arcs on the unit circle $\partial \mathbb{D}$,

$$
\operatorname{Arc}\left(\left[e^{i \theta_{1}}, e^{i \theta_{2}}\right]\right)=\left\{e^{i \theta} \in \partial \mathbb{D} \mid \theta_{1} \leq \theta \leq \theta_{2}\right\}, \quad \theta_{1} \in[0,2 \pi), \theta_{1} \leq \theta_{2} \leq \theta_{1}+2 \pi
$$

and similarly for open arcs and arcs open or closed at one endpoint (cf. (A.5)).

The principal new result of this paper then reads as follows. 
Theorem 4.2. Let $\alpha=\left\{\alpha_{k}\right\}_{k \in \mathbb{Z}}$ be a reflectionless sequence of $m \times m$ matrix-valued Verblunsky coefficients. Let $\mathbb{U}$ be the associated unitary CMV operator (2.10) on $\ell^{2}(\mathbb{Z})^{m}$ and suppose that the spectrum of $\mathbb{U}$ consists of a connected arc of $\partial \mathbb{D}$,

$$
\sigma(\mathbb{U})=\operatorname{Arc}\left(\left[e^{i \theta_{0}}, e^{i \theta_{1}}\right]\right)
$$

with $\theta_{0} \in[0,2 \pi), \theta_{0}<\theta_{1} \leq \theta_{0}+2 \pi$. Then $\alpha=\left\{\alpha_{k}\right\}_{k \in \mathbb{Z}}$ is of the form,

$$
\alpha_{k}=g^{k} a \gamma, \quad k \in \mathbb{Z},
$$

where

$$
g=-\exp \left(i\left(\theta_{0}+\theta_{1}\right) / 2\right), \quad a=\cos \left(\left(\theta_{1}-\theta_{0}\right) / 4\right),
$$

and $\gamma$ is some $k$-independent $m \times m$ unitary matrix.

Proof. First, note that in the special case $\sigma(\mathbb{U})=\partial \mathbb{D}$ the result follows from Theorem 4.1. Hence without loss of generality we will assume in the following that $\sigma(\mathbb{U}) \subsetneq \partial \mathbb{D}$, that is, $\theta_{1}-\theta_{0}<2 \pi$. Next, we proceed with the proof in two steps.

In our first step we find an explicit formula for the function $\Xi_{1,1}(\cdot, k)$ on $\partial \mathbb{D}$, $k \in \mathbb{Z}$. To understand the behavior of $\Xi_{1,1}(\cdot, k)$ it suffices by $(2.25)$ to study the behavior of the boundary values of $M_{1,1}(\cdot, k)$ on $\partial \mathbb{D}$.

We start by noting that Theorem A.2 (cf. (A.4)) implies that the Caratheodory matrix $M_{1,1}(\cdot, k)$ has purely imaginary (i.e., $\left.\operatorname{Re}\left(M_{1,1}(\cdot, k)\right)=0\right)$ boundary values $\mu_{0}$-a.e. on $\partial \mathbb{D} \backslash \operatorname{supp}\left(d \Omega_{1,1}(\cdot, k)\right)$. Moreover, it follows from (2.12) and Theorem 2.2 that

$$
\operatorname{supp}\left(d \Omega_{1,1}(\cdot, k)\right) \subseteq \operatorname{supp}(d \Omega(\cdot, k))=\sigma(\mathbb{U}), \quad k \in \mathbb{Z} .
$$

On the other hand, the reflectionless assumption, Theorem 3.2 (vii), and (4.9) imply that $M_{1,1}(\cdot, k)$ has strictly positive (i.e., $\left.M_{1,1}(\cdot, k)>0\right)$ boundary values $\mu_{0}$-a.e. on $\sigma_{\text {ess }}(\mathbb{U})=\sigma(\mathbb{U})$. By Theorem A.2 (cf. (A.4)) this implies that

$$
\sigma(\mathbb{U})=\sigma_{\mathrm{ess}}(\mathbb{U}) \subseteq \operatorname{supp}\left(d \Omega_{1,1}(\cdot, k)\right), \quad k \in \mathbb{Z} .
$$

Thus, it follows from (4.12) and (4.13) that $\sigma(\mathbb{U})=\operatorname{supp}\left(d \Omega_{1,1}(\cdot, k)\right), k \in \mathbb{Z}$. The same argument actually implies more, namely, that for any $x_{0} \in \mathbb{C}^{m}$ with $\left\|x_{0}\right\|_{\mathbb{C}^{m}}=1$, the scalar-valued Caratheodory function $m_{x_{0}}(\cdot, k)$ defined by

$$
\begin{aligned}
& m_{x_{0}}(z, k)=\left(x_{0}, M_{1,1}(z, k) x_{0}\right)_{\mathbb{C}^{m}}=\oint_{\partial \mathbb{D}} d \omega_{x_{0}}(\zeta, k) \frac{\zeta+z}{\zeta-z}, \\
& d \omega_{x_{0}}(\cdot, k)=d\left(x_{0}, \Omega_{1,1}(\cdot, k) x_{0}\right)_{\mathbb{C}^{m}}, \quad z \in \mathbb{D}, k \in \mathbb{Z},
\end{aligned}
$$

is purely imaginary $\mu_{0}$-a.e. on $\partial \mathbb{D} \backslash \sigma(\mathbb{U})$, strictly positive $\mu_{0}$-a.e. on $\sigma(\mathbb{U})$, and $\operatorname{supp}\left(d \omega_{x_{0}}(\cdot, k)\right)=\sigma(\mathbb{U})$ for all $k \in \mathbb{Z}$. Differentiating $-i m_{x_{0}}\left(e^{i \theta}, k\right)$ with respect to $\theta$ shows that $\operatorname{Im}\left(m_{x_{0}}(\cdot, k)\right)=-i m_{x_{0}}(\cdot, k)$ is monotone decreasing on $\partial \mathbb{D} \backslash \sigma(\mathbb{U})$,

$$
\frac{d}{d \theta}\left(-i m_{x_{0}}\left(e^{i \theta}, k\right)\right)=-\frac{1}{2} \int_{[0,2 \pi) \backslash\left[\theta_{0}, \theta_{1}\right]} \frac{d \omega_{x_{0}}\left(e^{i t}, k\right)}{\sin ^{2}((t-\theta) / 2)}<0, \quad \theta \in\left(\theta_{0}, \theta_{1}\right) .
$$

This implies that there exists a $\theta_{*}\left(x_{0}, k\right) \in\left[\theta_{1}, \theta_{0}+2 \pi\right]$ such that the exponential Herglotz representation for $m_{x_{0}}(\cdot, k)$,

$$
\ln \left[m_{x_{0}}(z, k)\right]=i \oint_{\partial \mathbb{D}} d \mu_{0}(\zeta) \xi_{k_{0}}(\zeta, k) \frac{\zeta+z}{\zeta-z}, \quad z \in \mathbb{D},
$$


yields the following form for the function $\xi_{x_{0}}(\zeta, k)=\lim _{r \uparrow 1} \operatorname{Im}\left[\ln \left(m_{x_{0}}(r \zeta, k)\right)\right]$,

$$
\xi_{x_{0}}(\zeta, k)=\left\{\begin{array}{ll}
0, & \zeta \in \operatorname{Arc}\left(\left(e^{i \theta_{0}}, e^{i \theta_{1}}\right)\right), \\
\pi / 2, & \zeta \in \operatorname{Arc}\left(\left(e^{i \theta_{1}}, e^{i \theta_{*}\left(x_{0}, k\right)}\right)\right), \\
-\pi / 2, & \zeta \in \operatorname{Arc}\left(\left(e^{i \theta_{*}\left(x_{0}, k\right)}, e^{i\left(\theta_{0}+2 \pi\right)}\right)\right)
\end{array} \quad \text { for } \mu_{0} \text {-a.e. } \zeta \in \partial \mathbb{D} .\right.
$$

Since by $(2.18) m_{x_{0}}(0, k)=\left\|x_{0}\right\|_{\mathbb{C}^{m}}^{2}=1, k \in \mathbb{Z}$, we compute using (4.17) and (4.18)

$$
\begin{aligned}
0 & =\ln \left[m_{x_{0}}(0, k)\right]=\oint_{\partial \mathbb{D}} d \mu_{0}(\zeta) \Xi_{1,1}(\zeta, k)=\frac{1}{4} \oint_{\theta_{1}}^{\theta_{*}\left(k, x_{0}\right)} d \theta-\frac{1}{4} \oint_{\theta_{*}\left(x_{0}, k\right)}^{\theta_{0}+2 \pi} d \theta \\
& =\frac{1}{4}\left[2 \theta_{*}\left(x_{0}, k\right)-\theta_{0}-\theta_{1}-2 \pi\right], \quad k \in \mathbb{Z}
\end{aligned}
$$

and hence

$$
\theta_{*}\left(x_{0}, k\right)=\frac{1}{2}\left(\theta_{0}+\theta_{1}\right)+\pi, \quad k \in \mathbb{Z},
$$

is in fact $\left(x_{0}, k\right)$-independent and denoted by $\theta_{*}$ in the following. As a result, $\xi_{x_{0}}(\cdot, k)=\xi(\cdot)$ in (4.18) and hence $m_{x_{0}}(\cdot, k)=m(\cdot)$ in (4.17) are also $\left(x_{0}, k\right)$ independent. Recalling (2.25), (4.14), and (4.18) we conclude that $M_{1,1}(\cdot, k)=$ $M_{1,1}(\cdot)$ and hence $\Xi_{1,1}(\cdot, k)=\Xi_{1,1}(\cdot)$ are $k$-independent and

$$
\Xi_{1,1}(\zeta)=\left\{\begin{array}{ll}
0, & \zeta \in \operatorname{Arc}\left(\left(e^{i \theta_{0}}, e^{i \theta_{1}}\right)\right), \\
\frac{\pi}{2} I_{m}, & \zeta \in \operatorname{Arc}\left(\left(e^{i \theta_{1}}, e^{i \theta_{*}}\right)\right), \\
-\frac{\pi}{2} I_{m}, & \zeta \in \operatorname{Arc}\left(\left(e^{i \theta_{*}}, e^{i\left(\theta_{0}+2 \pi\right)}\right)\right)
\end{array} \quad \text { for } \mu_{0} \text {-a.e. } \zeta \in \partial \mathbb{D} .\right.
$$

In our second step we use the above explicit form of the function $\Xi_{1,1}$ and the trace formulas obtained in Theorem 2.4 to derive various identities for Verblunsky coefficients $\alpha$ which will imply (4.10) and (4.11).

By (4.21) the following matrix

$$
\begin{aligned}
-i \oint_{\partial \mathbb{D}} d \mu_{0}(\zeta) \Xi_{1,1}(\zeta) \bar{\zeta} & =-i \oint_{\theta_{1}}^{\theta_{*}} \frac{\pi}{2} e^{-i t} \frac{d t}{2 \pi} I_{m}+i \oint_{\theta_{*}}^{\theta_{0}+2 \pi} \frac{\pi}{2} e^{-i t} \frac{d t}{2 \pi} I_{m} \\
& =-\frac{1}{4} e^{-i\left(\theta_{0}+\theta_{1}\right) / 2}\left(2+2 \cos \left(\left(\theta_{1}-\theta_{0}\right) / 2\right)\right) I_{m} \\
& =-e^{-i\left(\theta_{0}+\theta_{1}\right) / 2} \cos ^{2}\left(\left(\theta_{1}-\theta_{0}\right) / 4\right) I_{m}
\end{aligned}
$$

is a nonzero scalar multiple of the identity matrix $I_{m}$ since $0<\theta_{1}-\theta_{0}<2 \pi$. Hence, it follows from (2.37) that $\alpha_{k}$ is nonsingular, commutes with $\alpha_{k+1}^{*}$, and

$$
\alpha_{k+1}^{*} \alpha_{k}=\alpha_{k} \alpha_{k+1}^{*}=-e^{-i\left(\theta_{0}+\theta_{1}\right) / 2} \cos ^{2}\left(\left(\theta_{1}-\theta_{0}\right) / 4\right) I_{m}, \quad k \in \mathbb{Z} .
$$

Combining (2.5) with (4.23) one also gets

$$
\alpha_{k+1}^{*} \widetilde{\rho}_{k}=\rho_{k} \alpha_{k+1}^{*} \text { and } \widetilde{\rho}_{k} \alpha_{k-1}=\alpha_{k-1} \rho_{k}, \quad k \in \mathbb{Z} .
$$

Similarly, by (4.21)

$$
\begin{aligned}
-i \oint_{\partial \mathbb{D}} d \mu_{0}(\zeta) \Xi_{1,1}(\zeta) \bar{\zeta}^{2} & =-i \oint_{\theta_{1}}^{\theta_{*}} \frac{\pi}{2} e^{-2 i t} \frac{d t}{2 \pi} I_{m}+i \oint_{\theta_{*}}^{\theta_{0}+2 \pi} \frac{\pi}{2} e^{-2 i t} \frac{d t}{2 \pi} I_{m} \\
& =\frac{1}{4} e^{-i\left(\theta_{0}+\theta_{1}\right)}\left(1-\cos \left(\theta_{1}-\theta_{0}\right)\right) I_{m} \\
& =2 e^{-i\left(\theta_{0}+\theta_{1}\right)}\left(\cos ^{2}\left(\left(\theta_{1}-\theta_{0}\right) / 4\right)-\cos ^{4}\left(\left(\theta_{1}-\theta_{0}\right) / 4\right)\right) I_{m}
\end{aligned}
$$


is also a nonzero scalar multiple of the identity matrix $I_{m}$. Hence, (2.38) together with (4.23)-(4.25) implies

$$
\begin{aligned}
& \rho_{k+1} \alpha_{k+2}^{*} \widetilde{\rho}_{k+1} \alpha_{k}+\alpha_{k+1}^{*} \widetilde{\rho}_{k} \alpha_{k-1} \rho_{k}=\alpha_{k} \rho_{k+1} \alpha_{k+2}^{*} \widetilde{\rho}_{k+1}+\widetilde{\rho}_{k} \alpha_{k-1} \rho_{k} \alpha_{k+1}^{*} \\
& \quad=\alpha_{k} \rho_{k+1}^{2} \alpha_{k+2}^{*}+\alpha_{k-1} \rho_{k}^{2} \alpha_{k+1}^{*} \\
& \quad=2 e^{-i\left(\theta_{0}+\theta_{1}\right)}\left(\cos ^{2}\left(\left(\theta_{1}-\theta_{0}\right) / 4\right)-\cos ^{4}\left(\left(\theta_{1}-\theta_{0}\right) / 2\right)\right) I_{m}, \quad k \in \mathbb{Z} .
\end{aligned}
$$

Inserting $\rho_{k}^{2}=I_{m}-\alpha_{k}^{*} \alpha_{k}$ into the last equality of (4.26) and simplifying the expression using (4.23) yield

$$
\alpha_{k} \alpha_{k+2}^{*}+\alpha_{k-1} \alpha_{k+1}^{*}=2 e^{-i\left(\theta_{0}+\theta_{1}\right)} \cos ^{2}\left(\left(\theta_{1}-\theta_{0}\right) / 4\right) I_{m}, \quad k \in \mathbb{Z} .
$$

Multiplying both sides on the left and right by $\alpha_{k}^{*}$ and $\alpha_{k+1}$, respectively, and using (4.23) once again, imply

$$
\alpha_{k}^{*} \alpha_{k}+\alpha_{k+1}^{*} \alpha_{k+1}=2 \cos ^{2}\left(\left(\theta_{1}-\theta_{0}\right) / 4\right) I_{m}, \quad k \in \mathbb{Z} .
$$

Then (4.23) and (4.28) imply

$$
\alpha_{k+1}=-e^{i\left(\theta_{0}+\theta_{1}\right) / 2} \alpha_{k}, \quad k \in \mathbb{Z},
$$

since

$$
\begin{aligned}
& \left(\alpha_{k+1}+e^{i\left(\theta_{0}+\theta_{1}\right) / 2} \alpha_{k}\right)^{*}\left(\alpha_{k+1}+e^{i\left(\theta_{0}+\theta_{1}\right) / 2} \alpha_{k}\right) \\
& \quad=\alpha_{k}^{*} \alpha_{k}+\alpha_{k+1}^{*} \alpha_{k+1}+e^{i\left(\theta_{0}+\theta_{1}\right) / 2} \alpha_{k+1}^{*} \alpha_{k}+e^{-i\left(\theta_{0}+\theta_{1}\right) / 2} \alpha_{k}^{*} \alpha_{k+1} \\
& \quad=2 \cos ^{2}\left(\left(\theta_{1}-\theta_{0}\right) / 4\right) I_{m}-2 \cos ^{2}\left(\left(\theta_{1}-\theta_{0}\right) / 4\right) I_{m}=0, \quad k \in \mathbb{Z} .
\end{aligned}
$$

Inserting (4.29) into (4.23) yields

$$
\alpha_{k}^{*} \alpha_{k}=\cos ^{2}\left(\left(\theta_{1}-\theta_{0}\right) / 4\right) I_{m}, \quad k \in \mathbb{Z} .
$$

Finally, defining the unitary matrix $\gamma$ by $\gamma=\alpha_{0}\left(\alpha_{0}^{*} \alpha_{0}\right)^{-1 / 2}$ one obtains (4.10) and (4.11) from (4.29) and (4.31).

Remark 4.3. By Theorems 3.3 and 3.2 (ii) the unitary matrix $\gamma$ in (4.10) is a unitary invariant that preserves the reflectionless property, hence necessarily remains undetermined.

\section{Appendix A. Basic Facts on Caratheodory and Schur Functions}

In this appendix we summarize a few basic facts on matrix-valued Caratheodory and Schur functions used throughout this manuscript. (For the analogous case of matrix-valued Herglotz functions we refer to [38] and the extensive list of references therein.)

We denote by $\mathbb{D}$ and $\partial \mathbb{D}$ the open unit disk and the counterclockwise oriented unit circle in the complex plane $\mathbb{C}$,

$$
\mathbb{D}=\{z \in \mathbb{C}|| z \mid<1\}, \quad \partial \mathbb{D}=\{\zeta \in \mathbb{C}|| \zeta \mid=1\} .
$$

Moreover, we denote as usual $\operatorname{Re}(A)=\left(A+A^{*}\right) / 2$ and $\operatorname{Im}(A)=\left(A-A^{*}\right) /(2 i)$ for square matrices $A$ with complex-valued entries.

Definition A.1. Let $m \in \mathbb{N}$ and $F_{ \pm}, \Phi_{+}$, and $\Phi_{-}^{-1}$ be $m \times m$ matrix-valued analytic functions in $\mathbb{D}$.

(i) $F_{+}$is called a Caratheodory matrix if $\operatorname{Re}\left(F_{+}(z)\right) \geq 0$ for all $z \in \mathbb{D}$ and $F_{-}$is called an anti-Caratheodory matrix if $-F_{-}$is a Caratheodory matrix. 
(ii) $\Phi_{+}$is called a Schur matrix if $\left\|\Phi_{+}(z)\right\|_{\mathbb{C}^{m \times m}} \leq 1$, for all $z \in \mathbb{D}$. $\Phi_{-}$is called an anti-Schur matrix if $\Phi_{-}^{-1}$ is a Schur matrix.

Theorem A.2. Let $F$ be an $m \times m$ Caratheodory matrix, $m \in \mathbb{N}$. Then $F$ admits the Herglotz representation

$$
\begin{aligned}
& F(z)=i C+\oint_{\partial \mathbb{D}} d \Omega(\zeta) \frac{\zeta+z}{\zeta-z}, \quad z \in \mathbb{D}, \\
& C=\operatorname{Im}(F(0)), \quad \oint_{\partial \mathbb{D}} d \Omega(\zeta)=\operatorname{Re}(F(0)),
\end{aligned}
$$

where $d \Omega$ denotes a nonnegative $m \times m$ matrix-valued measure on $\partial \mathbb{D}$. The measure $d \Omega$ can be reconstructed from $F$ by the formula

$$
\Omega\left(\operatorname{Arc}\left(\left(e^{i \theta_{1}}, e^{i \theta_{2}}\right]\right)\right)=\lim _{\delta \downarrow 0} \lim _{r \uparrow 1} \frac{1}{2 \pi} \oint_{\theta_{1}+\delta}^{\theta_{2}+\delta} d \theta \operatorname{Re}(F(r \zeta)),
$$

where

$$
\operatorname{Arc}\left(\left(e^{i \theta_{1}}, e^{i \theta_{2}}\right]\right)=\left\{\zeta \in \partial \mathbb{D} \mid \theta_{1}<\theta \leq \theta_{2}\right\}, \quad \theta_{1} \in[0,2 \pi), \theta_{1}<\theta_{2} \leq \theta_{1}+2 \pi .
$$

Conversely, the right-hand side of equation (A.2) with $C=C^{*}$ and $d \Omega$ a finite nonnegative $m \times m$ matrix-valued measure on $\partial \mathbb{D}$ defines a Caratheodory matrix.

We note that additive nonnegative $m \times m$ matrices on the right-hand side of (A.2) can be absorbed into the measure $d \Omega$ since

$$
\oint_{\partial \mathbb{D}} d \mu_{0}(\zeta) \frac{\zeta+z}{\zeta-z}=1, \quad z \in \mathbb{D}
$$

where

$$
d \mu_{0}(\zeta)=\frac{d \theta}{2 \pi}, \quad \zeta=e^{i \theta}, \theta \in[0,2 \pi),
$$

denotes the normalized Lebesgue measure on the unit circle $\partial \mathbb{D}$.

Given a Caratheodory (resp., anti-Caratheodory) matrix $F_{+}$(resp. $F_{-}$) defined in $\mathbb{D}$ as in (A.2), one extends $F_{ \pm}$to all of $\mathbb{C} \backslash \partial \mathbb{D}$ by

$$
F_{ \pm}(z)=i C_{ \pm} \pm \oint_{\partial \mathbb{D}} d \Omega_{ \pm}(\zeta) \frac{\zeta+z}{\zeta-z}, \quad z \in \mathbb{C} \backslash \partial \mathbb{D}, C_{ \pm}=C_{ \pm}^{*}
$$

In particular,

$$
F_{ \pm}(z)=-F_{ \pm}(1 / \bar{z})^{*}, \quad z \in \mathbb{C} \backslash \overline{\mathbb{D}} .
$$

Of course, this continuation of $\left.F_{ \pm}\right|_{\mathbb{D}}$ to $\mathbb{C} \backslash \overline{\mathbb{D}}$, in general, is not an analytic continuation of $\left.F_{ \pm}\right|_{\mathbb{D}}$.

Next, given the functions $F_{ \pm}$defined in $\mathbb{C} \backslash \partial \mathbb{D}$ as in (A.8), we introduce the functions $\Phi_{ \pm}$by

$$
\Phi_{ \pm}(z)=\left[F_{ \pm}(z)-I_{m}\right]\left[F_{ \pm}(z)+I_{m}\right]^{-1}, \quad z \in \mathbb{C} \backslash \partial \mathbb{D} .
$$

We recall (cf., e.g., [72, p. 167]) that if $\pm \operatorname{Re}\left(F_{ \pm}\right) \geq 0$, then $\left[F_{ \pm} \pm I_{m}\right]$ is invertible. In particular, $\Phi_{+} \mid \mathbb{D}$ and $\left.\left[\Phi_{-}\right]^{-1}\right|_{\mathbb{D}}$ are Schur matrices (resp., $\left.\Phi_{-}\right|_{\mathbb{D}}$ is an anti-Schur matrix). Moreover,

$$
F_{ \pm}(z)=\left[I_{m}-\Phi_{ \pm}(z)\right]^{-1}\left[I_{m}+\Phi_{ \pm}(z)\right]
$$

and

$$
\Phi_{ \pm}(1 / \bar{z})=\left[\Phi_{ \pm}(z)^{*}\right]^{-1}, \quad z \in \mathbb{C} \backslash \partial \mathbb{D}
$$


M. ZINCHENKO

Acknowledgments. We are indebted to Fritz Gesztesy and Eric Ryckman for valuable comments and helpful discussions on this topic.

\section{REFERENCES}

[1] M. J. Ablowitz and J. F. Ladik, Nonlinear differential-difference equations, J. Math. Phys. 16, 598-603 (1975).

[2] M. J. Ablowitz and J. F. Ladik, Nonlinear differential-difference equations and Fourier analysis, J. Math. Phys. 17, 1011-1018 (1976).

[3] M. J. Ablowitz and J. F. Ladik, A nonlinear difference scheme and inverse scattering, Studies Appl. Math 55, 213-229 (1976).

[4] M. J. Ablowitz and J. F. Ladik, On the solution of a class of nonlinear partial difference equations, Studies Appl. Math. 57, 1-12 (1977).

[5] M. J. Ablowitz, B. Prinari, and A. D. Trubatch, Discrete and Continuous Nonlinear Schrödinger Systems, London Math. Soc. Lecture Note Series, Vol. 302, Cambridge University Press, Cambridge, 2004.

[6] N. I. Akhiezer, The Classical Moment Problem, Oliver \& Boyd., Edinburgh, 1965.

[7] E. D. Belokolos, F. Gesztesy, K. A. Makarov, and L. A. Sakhnovich, Matrix-valued generalizations of the theorems of Borg and Hochstadt, Evolution Equations, 1-34, Dekker, New York, 2003.

[8] Yu. M. Berezansky and M. E. Dudkin, The direct and inverse spectral problems for the block Jacobi type unitary matrices, Meth. Funct. Anal. Top. 11, 327-345 (2005).

[9] Yu. M. Berezansky and M. E. Dudkin, The complex moment problem and direct and inverse spectral problems for the block Jacobi type bounded normal matrices, Meth. Funct. Anal. Top. 12, 1-31 (2006).

[10] G. Borg, Eine Umkehrung der Sturm-Liouvilleschen Eigenwertaufgabe, Acta Math. 78, 1-96 (1946).

[11] O. Bourget, J. S. Howland, and A. Joye, Spectral analysis of unitary band matrices, Commun. Math. Phys. 234, 191-227 (2003).

[12] A. Bunse-Gerstner and L. Elsner, Schur parameter pencils for the solution of unitary eigenproblem, Lin. Algebra Appl. 154/156, 741-778 (1991).

[13] M. J. Cantero, L. Moral, and L. Velázquez, Five-diagonal matrices and zeros of orthogonal polynomials on the unit circle, Lin. Algebra Appl. 362, 29-56 (2003).

[14] R. W. Carey, A unitary invariant for pairs of self-adjoint operators, J. reine angew. Math. 283, 294-312 (1976).

[15] S. Clark and F. Gesztesy, Weyl-Titchmarsh M-function asymptotics and Borg-type theorems for Dirac operators, Trans. Amer. Math. Soc. 354, 3475-3534 (2002).

[16] S. Clark, F. Gesztesy, H. Holden, and B. M. Levitan, Borg-type theorems for matrix-valued Schrödinger operators, J. Diff. Eqs. 167, 181-210 (2000).

[17] S. Clark, F. Gesztesy, and W. Renger, Borg-type theorems for matrix-valued Jacobi and Dirac finite difference operators, J. Diff. Eq. 219, 144-182 (2005).

[18] S. Clark, F. Gesztesy, and M. Zinchenko, Weyl-Titchmarsh theory and Borg-Marchenkotype uniqueness results for CMV operators with matrix-valued Verblunsky coefficients, Oper. Matrices 1, No. 4, 535-592 (2007).

[19] W. Craig, The trace formula for Schrödinger operators on the line, Commun. Math. Phys. 126, 379-407 (1989).

[20] D. Damanik, R. Killip, B. Simon, Perturbations of orthogonal polynomials with periodic recursion coefficients, preprint.

[21] D. Damanik, A. Pushnitski, B. Simon, The analytic theory of matrix orthogonal polynomials, Surv. Approx. Th. 4, 1-85 (2008).

[22] P. Deift and B. Simon, Almost periodic Schrödinger operators III. The absolutely continuous spectrum in one dimension, Commun. Math. Phys. 90, 389-411 (1983).

[23] B. Després, The Borg theorem for the vectorial Hill's equation, Inverse Probl. 11, 97-121 (1995). 
[24] H. Flaschka, Discrete and periodic illustrations of some aspects of the inverse method, in Dynamical Systems, Theory and Applications, J. Moser (ed.), Lecture Notes In Physics, Vol. 38, Springer Verlag, Berlin, 1975, p. 441-466.

[25] J. S. Geronimo, F. Gesztesy, H. Holden, Algebro-geometric solutions of the Baxter-Szegó difference equation, Commun. Math. Phys. 258, 149-177 (2005).

[26] J. S. Geronimo and R. Johnson, Rotation number associated with difference equations satisfied by polynomials orthogonal on the unit circle, J. Diff. Eqs. 132, 140-178 (1996).

[27] J. S. Geronimo and R. Johnson, An inverse problem associated with polynomials orthogonal on the unit circle, Commun. Math. Phys. 193, 125-150 (1998).

[28] J. S. Geronimo and A. Teplyaev, A difference equation arising from the trigonometric moment problem having random reflection coefficients-an operator theoretic approach, J. Funct. Anal. 123, 12-45 (1994).

[29] J. Geronimus, On the trigonometric moment problem, Ann. Math. 47, 742-761 (1946).

[30] Ya. L. Geronimus, Polynomials orthogonal on a circle and their applications, Commun. Soc. Mat. Kharkov 15, 35-120 (1948); Amer. Math. Soc. Transl. (1) 3, 1-78 (1962).

[31] Ya. L. Geronimus, Orthogonal Polynomials, Consultants Bureau, New York, 1961.

[32] F. Gesztesy and H. Holden, Soliton Equations and Their Algebro-Geometric Solutions. Volume II: (1+1)-Dimensional Discrete Models, Cambridge Studies in Adv. Math., Cambridge University Press, Cambridge, to appear.

[33] F. Gesztesy, H. Holden, J. Michor, and G. Teschl The AblowitzLadik hierarchy revisited, In Proceedings of the conference on Operator Theory, Analysis in Mathematical Physics OTAMP 2006, J. Janas, P. Kurasov, A. Laptev, S. Naboko, and G. Stolz (eds.), Operator Theory: Advances and Applications, Birkhäuser, Basel (to appear).

[34] F. Gesztesy, H. Holden, J. Michor, and G. Teschl, Algebro-geometric finite-band solutions of the Ablowitz-Ladik hierarchy, Int. Math. Res. Notices, 2007, rnm082, 55 pages.

[35] F. Gesztesy, H. Holden, J. Michor, and G. Teschl The algebro-geometric initial value problem for the AblowitzLadik hierarchy, preprint.

[36] F. Gesztesy, M. Krishna, and G. Teschl, On isospectral sets of Jacobi operators, Commun. Math. Phys. 181, 631-645 (1996)

[37] F. Gesztesy and B. Simon, The $\xi$ function, Acta Math. 176, 49-71 (1996).

[38] F. Gesztesy and E. Tsekanovskii, On matrix-valued Herglotz functions, Math. Nachr. 218, 61-138 (2000).

[39] F. Gesztesy and M. Zinchenko, A Borg-type theorem associated with orthogonal polynomials on the unit circle, J. London Math. Soc. (2) 74, No. 3, 757-777 (2006).

[40] F. Gesztesy and M. Zinchenko, Weyl-Titchmarsh theory for CMV operators associated with orthogonal polynomials on the unit circle, J. Approx. Th. 139, 172-213 (2006).

[41] R. Giachetti and R. A. Johnson, Spectral theory of second-order almost periodic differential operators and its relation to classes of nonlinear evolution equations, Nuovo Cim. 82B, $125-168$ (1984).

[42] R. Giachetti and R. A. Johnson, The Floquet exponent for two-dimensional linear systems with bounded coefficients, J. Math. pures et appl. 65, 93-117 (1986).

[43] L. Golinskii and P. Nevai, Szegö difference equations, transfer matrices and orthogonal polynomials on the unit circle, Commun. Math. Phys. 223, 223-259 (2001).

[44] R. A. Johnson, The recurrent Hill's equation, J. Diff. Eqs. 46, 165-193 (1982).

[45] R. Killip and I. Nenciu, CMV: the unitary analogue of Jacobi matrices, Comm. Pure Appl. Math. 60, No. 8, 1148-1188 (2007).

[46] S. Kotani, Ljapunov indices determine absolutely continuous spectra of stationary random one-dimensional Schrödinger operators, in Stochastic Analysis, K. Itǒ (ed.), North-Holland, Amsterdam, 1984, pp. 225-247.

[47] S. Kotani, One-dimensional random Schrödinger operators and Herglotz functions, in Probabilistic Methods in Mathematical Physics, K. Itǒ and N. Ikeda (eds.), Academic Press, New York, 1987, pp. 219-250.

[48] S. Kotani and M. Krishna, Almost periodicity of some random potentials, J. Funct. Anal. 78, 390-405 (1988)

[49] S. Kotani and B. Simon, Stochastic Schrödinger operators and Jacobi matrices on the strip, Comm. Math. Phys. 119, No. 3, 403-429 (1988).

[50] M. G. Krein, On a generalization of some investigations of G. Szegő, V. Smirnoff, and A. Kolmogoroff, Dokl. Akad. Nauk SSSR 46, 91-94 (1945). (Russian). 
[51] L.-C. Li, Some remarks on CMV matrices and dressing orbits, Int. Math. Res. Notices 40, 2437-2446 (2005).

[52] A. L. Lukashov, Circular parameters of polynomials orthogonal on several arcs of the unit circle, Sbornik Math. 195, 1639-1663 (2004).

[53] M. M. Malamud, Similarity of Volterra operators and related questions of the theory of differential equations of fractional order, Trans. Moscow Math. Soc. 55, 57-122 (1994).

[54] M. M. Malamud, Borg type theorems for first-order systems on a finite interval, Funct. Anal. Appl. 33, 64-68 (1999).

[55] P. D. Miller, N. M. Ercolani, I. M. Krichever, and C. D. Levermore, Finite genus solutions to the Ablowitz-Ladik equations, Comm. Pure Appl. Math. 4, 1369-1440 (1995).

[56] I. Nenciu, Lax pairs for the Ablowitz-Ladik system via orthogonal polynomials on the unit circle, Int. Math. Res. Notices 2005:11, 647-686 (2005).

[57] I. Nenciu, Lax Pairs for the Ablowitz-Ladik System via Orthogonal Polynomials on the Unit Circle, Ph.D. Thesis, Caltech, 2005.

[58] I. Nenciu, CMV matrices in random matrix theory and integrable systems: a survey, J. Phys. A 39, 8811-8822 (2006).

[59] F. Peherstorfer and P. Yuditskii, Asymptotic behavior of polynomials orthonormal on a homogeneous set, J. Analyse Math. 89, 113-154 (2003).

[60] B. Simon, Analogs of the m-function in the theory of orthogonal polynomials on the unit circle, J. Comp. Appl. Math. 171, 411-424 (2004).

[61] B. Simon, Orthogonal Polynomials on the Unit Circle, Part 1: Classical Theory, Part 2: Spectral Theory, AMS Colloquium Publication Series, Vol. 54, Providence, R.I., 2005.

[62] B. Simon, OPUC on one foot, Bull. Amer. Math. Soc. 42, 431-460 (2005).

[63] B. Simon, CMV matrices: Five years later, J. Comp. Appl. Math. 208, 120-154 (2007).

[64] M. Sodin and P. Yuditskii, Almost periodic Sturm-Liouville operators with Cantor homogeneous spectrum, Comment. Math. Helvetici 70, 639-658 (1995).

[65] M. Sodin and P. Yuditskii, Almost periodic Jacobi matrices with homogeneous spectrum, infinite dimensional Jacobi inversion, and Hardy spaces of character-automorphic functions, J. Geom. Anal. 7, 387-435 (1997).

[66] G. Teschl, Trace formulas and inverse spectral theory for Jacobi operators, Commun. Math. Phys. 196, 175-202 (1998).

[67] G. Teschl, Jacobi Operators and Completely Integrable Nonlinear Lattices, Mathematical Surveys and Monographs, 72, American Mathematical Society, (2000).

[68] R. J. Schilling, A systematic approach to the soliton equations of a discrete eigenvalue problem, J. Math. Phys. 30, 1487-1501 (1989).

[69] G. Szegö, Beiträge zur Theorie der Toeplitzschen Formen I, Math. Z. 6, 167-202 (1920).

[70] G. Szegö, Beiträge zur Theorie der Toeplitzschen Formen II, Math. Z. 9, 167-190 (1921).

[71] G. Szegö, Orthogonal Polynomials, Amer Math. Soc. Colloq. Publ., Vol. 23, Amer. Math. Soc., Providence, R.I., 1978.

[72] B. Sz.-Nagy and C. Foiaş, Harmonic Analysis of Operators on Hilbert Space, North-Holland, Amsterdam, 1970

[73] V. E. Vekslerchik, Finite genus solutions for the Ablowitz-Ladik hierarchy, J. Phys. A 32, 4983-4994 (1998).

[74] S. Verblunsky, On positive harmonic functions: A contribution to the algebra of Fourier series, Proc. London Math. Soc. (2) 38, 125-157 (1935).

[75] S. Verblunsky, On positive harmonic functions (second paper), Proc. London Math. Soc. (2) 40, 290-320 (1936).

[76] D. S. Watkins, Some perspectives on the eigenvalue problem, SIAM Rev. 35, 430-471 (1993).

Department of Mathematics, California institute of Technology, Pasadena, CA 91125, USA

E-mail address: maxim@caltech.edu

$U R L:$ http://www.math.caltech.edu/ ${ }^{\sim m a x i m}$ 